This item was submitted to Loughborough's Research Repository by the author.

Items in Figshare are protected by copyright, with all rights reserved, unless otherwise indicated.

\title{
Integrable systems in four dimensions associated with six-folds in $\operatorname{Gr}(4,6)$
}

PLEASE CITE THE PUBLISHED VERSION

https://doi.org/10.1093/imrn/rnx308

PUBLISHER

Oxford University Press @ The authors

\section{VERSION}

AM (Accepted Manuscript)

\section{PUBLISHER STATEMENT}

This work is made available according to the conditions of the Creative Commons Attribution-NonCommercialNoDerivatives 4.0 International (CC BY-NC-ND 4.0) licence. Full details of this licence are available at: https://creativecommons.org/licenses/by-nc-nd/4.0/

\section{LICENCE}

CC BY-NC-ND 4.0

\section{REPOSITORY RECORD}

Doubrov, B., Evgeny Ferapontov, B. Kruglikov, and Vladimir Novikov. 2019. "Integrable Systems in Four Dimensions Associated with Six-folds in $\operatorname{Gr}(4,6)$ ”. figshare. https://hdl.handle.net/2134/27771. 


\section{Integrable systems in 4D associated with sixfolds in $\mathbf{G r}(4,6)$}

B. Doubrov ${ }^{1}$, E.V. Ferapontov ${ }^{2}$, B. Kruglikov ${ }^{3}$, V.S. Novikov ${ }^{2}$

${ }^{1}$ Department of Mathematical Physics

Faculty of Applied Mathematics

Belarussian State University

Nezavisimosti av. 4, 220030 Minsk, Belarus

${ }^{2}$ Department of Mathematical Sciences

Loughborough University

Loughborough, Leicestershire LE11 3TU

United Kingdom

${ }^{3}$ Department of Mathematics and Statistics

Faculty of Science and Technology

UiT the Arctic University of Norway

Troms $\varnothing$ 90-37, Norway

$$
\text { e-mails: }
$$

doubrov@islc.org

E.V.Ferapontov@lboro.ac.uk

boris.kruglikov@uit.no

V.Novikov@lboro.ac.uk 


\begin{abstract}
Let $\mathbf{G r}(d, n)$ be the Grassmannian of $d$-dimensional linear subspaces of an $n$-dimensional vector space $V$. A submanifold $X \subset \mathbf{G r}(d, n)$ gives rise to a differential system $\Sigma(X)$ that governs $d$-dimensional submanifolds of $V$ whose Gaussian image is contained in $X$. We investigate a special case of this construction where $X$ is a sixfold in $\mathbf{G r}(4,6)$. The corresponding system $\Sigma(X)$ reduces to a pair of first-order PDEs for 2 functions of 4 independent variables. Equations of this type arise in self-dual Ricci-flat geometry. Our main result is a complete description of integrable systems $\Sigma(X)$. These naturally fall into two subclasses.
\end{abstract}

- Systems of Monge-Ampère type. The corresponding sixfolds $X$ are codimension 2 linear sections of the Plücker embedding $\mathbf{G r}(4,6) \hookrightarrow \mathbb{P}^{14}$.

- General linearly degenerate systems. The corresponding sixfolds $X$ are the images of quadratic maps $\mathbb{P}^{6} \rightarrow \mathbf{G r}(4,6)$ given by a version of the classical construction of Chasles.

We prove that integrability is equivalent to the requirement that the characteristic variety of system $\Sigma(X)$ gives rise to a conformal structure which is self-dual on every solution. In fact, all solutions carry hyper-Hermitian geometry.

MSC: 37K10, 37K25, 53A30, 53A40, 53B15, 53B25, 53B50, $53 \mathrm{Z} 05$.

Keywords: Submanifold of the Grassmannian, Dispersionless Integrable System, Hydrodynamic Reduction, Self-dual Conformal Structure, Monge-Ampère System, Dispersionless Lax Pair, Linear Degeneracy.

\title{
Contents
}

1 Introduction $\quad 3$

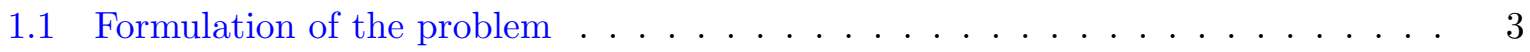

1.2 Non-degeneracy, conformal structure and self-duality . . . . . . . . . . 4

1.3 Dispersionless integrability in $4 \mathrm{D} \ldots \ldots \ldots \ldots \ldots$

1.4 Equivalence group $\mathbf{S L}(6) \ldots \ldots \ldots \ldots \ldots \ldots$

1.5 Linearly degenerate systems . . . . . . . . . . . . . . . . . 6

1.6 Summary of the main results $\ldots \ldots \ldots \ldots \ldots \ldots$

2 Examples and classification results $\quad 8$

2.1 Monge-Ampère systems $\ldots \ldots \ldots \ldots \ldots \ldots$

2.2 Linearisable systems . . . . . . . . . . . . . . . . . . . . . . . 9

2.3 Systems associated with quadratic maps $\mathbb{P}^{6} \rightarrow \mathbf{G r}(4,6) \ldots \ldots \ldots$

2.4 Symmetries of general linearly degenerate systems . . . . . . . . . . . . 13

3 Proofs of the main results 14

3.1 Action of the equivalence group . . . . . . . . . . . . . 14

3.2 Prolongation of the conditions of linear degeneracy . . . . . . . . . 15

3.3 Proof of Theorem $1 \ldots \ldots \ldots \ldots \ldots \ldots$

4 Concluding remarks $\quad 20$ 


\section{Introduction}

\subsection{Formulation of the problem}

Let $u(\mathbf{x})$ and $v(\mathbf{x})$ be functions of the 4 independent variables $\mathbf{x}=\left(x^{1}, \ldots, x^{4}\right)$. In this paper we investigate integrability of first-order systems of the form

$$
F\left(u_{1}, \ldots, u_{4}, v_{1}, \ldots, v_{4}\right)=0, \quad H\left(u_{1}, \ldots, u_{4}, v_{1}, \ldots, v_{4}\right)=0,
$$

where $F$ and $H$ are (nonlinear) functions of the partial derivatives $u_{i}=\frac{\partial u}{\partial x^{i}}, v_{i}=\frac{\partial v}{\partial x^{i}}$. The geometry behind systems (1) is as follows. Let $V$ be a 6 -dimensional vector space with coordinates $x^{1}, \ldots, x^{4}, u, v$. Solutions to system (1) correspond to 4-dimensional submanifolds of $V$ defined as $u=u(\mathbf{x}), v=v(\mathbf{x})$. Their 4-dimensional tangent spaces, specified by the equations $d u=u_{i} d x^{i}, d v=v_{i} d x^{i}$, are parametrised by $2 \times 4$ matrices

$$
U=\left(\begin{array}{lll}
u_{1} & \ldots & u_{4} \\
v_{1} & \ldots & v_{4}
\end{array}\right)
$$

whose entries are restricted by equations (1). Thus, equations (1) can be interpreted as the defining equations of a sixfold $X$ in the Grassmannian $\mathbf{G r}(4,6)$. Solutions to system (1) correspond to 4-dimensional submanifolds of $V$ whose Gaussian images (tangent spaces translated to the origin) are contained in $X$. There exist two types of integrable systems (1).

Systems of Monge-Ampère type have the form

$$
\begin{aligned}
& a^{i j}\left(u_{i} v_{j}-u_{j} v_{i}\right)+b^{i} u_{i}+c^{i} v_{i}+m=0, \\
& \alpha^{i j}\left(u_{i} v_{j}-u_{j} v_{i}\right)+\beta^{i} u_{i}+\gamma^{i} v_{i}+\mu=0,
\end{aligned}
$$

where each equation is a constant-coefficient linear combination of the minors of $U$. These systems were introduced in [2] in the context of 'complete exceptionality'. Geometrically, the associated sixfolds $X$ are linear sections of the Plücker embedding $\operatorname{Gr}(4,6) \hookrightarrow \mathbb{P}^{14}$. A typical example is the system

$$
u_{2}-v_{1}=0, \quad u_{3} v_{4}-u_{4} v_{3}-1=0,
$$

which reduces to the first heavenly equation of Plebanski [24], $w_{13} w_{24}-w_{14} w_{23}-1=0$, under the substitution $w_{1}=u, w_{2}=v$. It governs self-dual Ricci-flat 4-manifolds; see Section 2.1 for further details on Monge-Ampère systems.

General linearly degenerate systems correspond to sixfolds $X$ resulting as images of quadratic maps $\mathbb{P}^{6} \rightarrow \mathbf{G r}(4,6)$ (we refer to [7] for a discussion of the concept of linear degeneracy, see also Section 1.5). As an example, let us consider the system

$$
\alpha u_{2} v_{1}-u_{1} v_{2}=0, \quad u_{4} v_{1}-u_{1} v_{3}=0
$$

$\alpha \neq 0,1$ is a parameter. Note that this system does not belong to the Monge-Ampère class (2). The elimination of $v$ leads to the second-order equation for $u$,

$$
\left(\partial_{3}-\partial_{4}\right) \frac{u_{2}}{u_{1}}=\left(\alpha^{-1}-1\right) \partial_{2} \frac{u_{4}}{u_{1}},
$$

here $\partial_{i}=\partial_{x^{i}}$. Similarly, the elimination of $u$ leads to the second-order equation for $v$,

$$
\left(\partial_{4}-\partial_{3}\right) \frac{v_{2}}{v_{1}}=(\alpha-1) \partial_{2} \frac{v_{3}}{v_{1}} .
$$


Thus, one can speak of a four-dimensional Bäcklund transformation. This example can be viewed as a $4 \mathrm{D}$ generalisation of the Bäcklund transformation for the Veronese web equation constructed in [28]. We refer to Section 2.3 for further examples and classification results.

The main goal of this paper is to prove that systems of the above two types exhaust the list of non-degenerate integrable systems (1).

\subsection{Non-degeneracy, conformal structure and self-duality}

We will assume that system (1) is non-degenerate in the sense that the corresponding characteristic variety,

$$
\operatorname{det}\left[\sum_{i=1}^{4} p_{i}\left(\begin{array}{ll}
F_{u_{i}} & F_{v_{i}} \\
H_{u_{i}} & H_{v_{i}}
\end{array}\right)\right]=0,
$$

defines an irreducible quadric of rank 4 . This is the case for all examples of physical/geometric relevance. Explicitly, the characteristic variety can be represented in the form $g^{i j} p_{i} p_{j}=0$ where

$$
g^{i j}=\frac{1}{2}\left(F_{u_{i}} H_{v_{j}}+F_{u_{j}} H_{v_{i}}-F_{v_{i}} H_{u_{j}}-F_{v_{j}} H_{u_{i}}\right) .
$$

The characteristic variety gives rise to the conformal structure $g=g_{i j} d x^{i} d x^{j}$ where $g_{i j}$ is the inverse matrix of $g^{i j}$; note that non-degeneracy is equivalent to $\operatorname{det} g \neq 0$. Let $[g]$ denote the corresponding conformal class. Remarkably, integrability of system (1) has a natural interpretation in terms of the conformal geometry of $[g]$. In $4 \mathrm{D}$, the key invariant of a conformal structure is its Weyl tensor $W$. It has self-dual and anti-self-dual parts,

$$
W_{+}=\frac{1}{2}(W+* W) \quad \text { and } \quad W_{-}=\frac{1}{2}(W-* W),
$$

respectively. Here the Hodge star operator is defined as $* W_{j k l}^{i}=\frac{1}{2} \sqrt{\operatorname{det} g} g^{i a} g^{b c} \epsilon_{a j b d} W_{c k l}^{d}$. A conformal structure is said to be self-dual if, with a proper choice of orientation, we have

$$
W_{-}=0 .
$$

The integrability of conditions of self-duality by the twistor construction is due to Penrose [23], see also [10] for a direct demonstration. We will prove in Section 3 that integrability of $4 \mathrm{D}$ equations (1) is equivalent to the requirement that the conformal structure $[g]$ defined by the characteristic variety must be self-dual on every solution. Thus, solutions to integrable systems carry integrable conformal geometry. More precisely, with a suitable choice of orientation, it will be shown that the conditions of self-duality, $W_{-}=0$, lead to Monge-Ampère systems. Similarly, the conditions of anti-self-duality, $W_{+}=0$, characterise general linearly degenerate systems associated with quadratic maps $\mathbb{P}^{6} \rightarrow \mathbf{G r}(4,6)$. The intersection of these two classes consists of linearisable systems characterised by the conformal flatness of $g$.

For example, the conformal structure of system (3) is given by

$$
g=u_{3} d x^{1} d x^{3}+u_{4} d x^{1} d x^{4}+v_{3} d x^{2} d x^{3}+v_{4} d x^{2} d x^{4} .
$$

A direct calculation shows that $[g]$ is self-dual on every solution, which means that (4) holds identically modulo (3). System (3) possesses the Lax representation $[X, Y]=0$ where $X, Y$ are parameter-dependent vector fields,

$$
X=u_{3} \partial_{4}-u_{4} \partial_{3}+\lambda \partial_{1}, \quad Y=-v_{3} \partial_{4}+v_{4} \partial_{3}-\lambda \partial_{2},
$$


$\partial_{i}=\partial_{x^{i}}$. Projecting integral surfaces of the distribution spanned by $X, Y$ from the extended space of variables $\mathbf{x}, \lambda$ (correspondence space) to the space of independent variables $\mathbf{x}$ one obtains a three-parameter family of totally null surfaces ( $\alpha$-surfaces) of the conformal structure $[g]$. According to [23], the existence of such surfaces is necessary and sufficient for self-duality. We refer to $[1,20,21]$ for a novel version of the inverse scattering transform based on commuting parameter-dependent vector fields.

\subsection{Dispersionless integrability in $4 \mathrm{D}$}

Integrability of multi-dimensional dispersionless PDEs can be approached based on the method of hydrodynamic reductions $[17,12,11,13]$. In the most general set-up (for definiteness, we restrict to the $4 \mathrm{D}$ case), it applies to quasilinear systems of the form

$$
A_{1}(\mathbf{u}) \mathbf{u}_{1}+A_{2}(\mathbf{u}) \mathbf{u}_{2}+A_{3}(\mathbf{u}) \mathbf{u}_{3}+A_{4}(\mathbf{u}) \mathbf{u}_{4}=0,
$$

where $\mathbf{u}=\left(u^{1}, \ldots, u^{m}\right)^{t}$ is an $m$-component column vector of the dependent variables, $\mathbf{u}_{i}=\frac{\partial \mathbf{u}}{\partial x^{i}}$, and $A_{i}$ are $l \times m$ matrices where the number $l$ of equations is allowed to exceed the number $m$ of unknowns. Note that nonlinear system (1) can be brought to quasilinear form (5) by choosing $u_{i}, v_{i}$ as the new dependent variables and writing out all possible consistency conditions among them, see Section 3. The method of hydrodynamic reductions consists of seeking multi-phase solutions in the form

$$
\mathbf{u}=\mathbf{u}\left(R^{1}, \ldots, R^{N}\right)
$$

where the phases $R^{i}(\mathbf{x})$, whose number $N$ is allowed to be arbitrary, are required to satisfy a triple of consistent $(1+1)$-dimensional systems

$$
R_{x^{2}}^{i}=\mu^{i}(R) R_{x^{1}}^{i}, \quad R_{x^{3}}^{i}=\eta^{i}(R) R_{x^{1}}^{i}, \quad R_{x^{4}}^{i}=\lambda^{i}(R) R_{x^{1}}^{i},
$$

known as systems of hydrodynamic type. The corresponding characteristic speeds must satisfy the commutativity conditions [27],

$$
\frac{\partial_{j} \mu^{i}}{\mu^{j}-\mu^{i}}=\frac{\partial_{j} \eta^{i}}{\eta^{j}-\eta^{i}}=\frac{\partial_{j} \lambda^{i}}{\lambda^{j}-\lambda^{i}}
$$

here $i \neq j, \partial_{j}=\partial_{R^{j}}$. Multi-phase solutions of this type originate from gas dynamics, and are known as nonlinear interactions of planar simple waves. Equations (6) are said to define an $N$-component hydrodynamic reduction of the original system (5). System (5) is said to be integrable if, for every $N$, it possesses infinitely many $N$-component hydrodynamic reductions parametrised by $2 N$ arbitrary functions of one variable [13]. This requirement imposes strong constraints (integrability conditions) on the matrix elements of $A_{i}(\mathbf{u})$, see Section 3 for details.

The method of hydrodynamic reductions has been successfully applied to a whole range of systems in $3 \mathrm{D}$, leading to extensive classification results. The corresponding submanifolds $X$ are generally transcendental, parametrised by generalised hypergeometric functions [22]. The results of this paper are based on a direct application of the method of hydrodynamic reductions to $4 \mathrm{D}$ systems of type (1). The 4D situation turns out to be far more restrictive, in particular, the integrability conditions force $X$ to be algebraic. 


\subsection{Equivalence group SL(6)}

All constructions described in the previous sections are equivariant with respect to the group $\mathbf{S L}(6)$ acting by linear transformations on the space $V$ with coordinates $x^{1}, \ldots, x^{4}, u, v$. The extension of this action to $\mathbf{G r}(4,6)$ is given by the formula

$$
U \rightarrow(A U+B)(C U+D)^{-1}
$$

where $A, B, C, D$ are $2 \times 2,2 \times 4,4 \times 2$ and $4 \times 4$ matrices, respectively; note that the extended action is no longer linear. Transformation law (8) suggests that the action of SL(6) preserves the class of equations (1). Furthermore, transformations (8) preserve the integrability, so that SL(6) can be viewed as a natural equivalence group of the problem: all our classification results will be formulated modulo this equivalence. In coordinates $u_{i}, v_{i}$, the infinitesimal generators corresponding to equivalence transformations (8) are as follows:

8 translations:

$$
\mathbf{U}_{i}=\frac{\partial}{\partial u_{i}}, \quad \mathbf{V}_{i}=\frac{\partial}{\partial v_{i}},
$$

19 linear generators (note the relation $\sum \mathbf{X}_{i i}=\mathbf{L}_{11}+\mathbf{L}_{22}$ ):

$$
\mathbf{X}_{i j}=u_{i} \frac{\partial}{\partial u_{j}}+v_{i} \frac{\partial}{\partial v_{j}}, \quad \mathbf{L}_{11}=u_{k} \frac{\partial}{\partial u_{k}}, \quad \mathbf{L}_{12}=u_{k} \frac{\partial}{\partial v_{k}}, \quad \mathbf{L}_{21}=v_{k} \frac{\partial}{\partial u_{k}}, \quad \mathbf{L}_{22}=v_{k} \frac{\partial}{\partial v_{k}} .
$$

8 projective generators:

$$
\mathbf{P}_{i}=u_{i} u_{k} \frac{\partial}{\partial u_{k}}+v_{i} u_{k} \frac{\partial}{\partial v_{k}}, \quad \mathbf{Q}_{i}=u_{i} v_{k} \frac{\partial}{\partial u_{k}}+v_{i} v_{k} \frac{\partial}{\partial v_{k}} .
$$

Let us represent system (1) in evolutionary form,

$$
u_{4}=f\left(u_{1}, u_{2}, u_{3}, v_{1}, v_{2}, v_{3}\right), \quad v_{4}=h\left(u_{1}, u_{2}, u_{3}, v_{1}, v_{2}, v_{3}\right),
$$

and consider the induced action of the equivalence group $\mathbf{S L}(6)$ on the space $J^{1}\left(\mathbb{R}^{6}, \mathbb{R}^{2}\right)$ of 1-jets of functions $f, h$ of variables $u_{1}, u_{2}, u_{3}, v_{1}, v_{2}, v_{3}$. This is a 20 -dimensional space with coordinates $u_{i}, v_{i}, f, h, f_{u_{i}}, f_{v_{i}}, h_{u_{i}}, h_{v_{i}}, i=1,2,3$. One can show that the action of $\mathbf{S L}(6)$ on $J^{1}\left(\mathbb{R}^{6}, \mathbb{R}^{2}\right)$ has a unique Zariski open orbit (its complement consists of 1-jets of degenerate systems), see Section 3.1. This property allows one to assume that all sporadic factors depending on first-order derivatives of $f$ and $h$ that arise in the process of Gaussian elimination in the proofs of our main results in Section 3, are nonzero. This considerably simplifies the arguments by eliminating unessential branching. Furthermore, in the verification of polynomial identities involving firstand second-order partial derivatives of $f$ and $h$ one can, without any loss of generality, give the first-order derivatives any 'generic' numerical values: this often renders otherwise impossible computations manageable.

\subsection{Linearly degenerate systems}

The definition of linear degeneracy is inductive: a multi-dimensional system is said to be linearly degenerate (completely exceptional [2]) if such are all its traveling wave reductions to two dimensions. Thus, it is sufficient to define this concept in the $2 \mathrm{D}$ case,

$$
u_{2}=f\left(u_{1}, v_{1}\right), \quad v_{2}=h\left(u_{1}, v_{1}\right) .
$$


Setting $u_{1}=a, v_{1}=p$ and differentiating by $x^{1}$ one can rewrite this system in two-component quasilinear form,

$$
a_{2}=f(a, p)_{1}, \quad p_{2}=h(a, p)_{1},
$$

or, in matrix notation,

$$
\left(\begin{array}{l}
a \\
p
\end{array}\right)_{2}=A\left(\begin{array}{c}
a \\
p
\end{array}\right)_{1}, \quad A=\left(\begin{array}{ll}
f_{a} & f_{p} \\
h_{a} & h_{p}
\end{array}\right) .
$$

Recall that the matrix $A$ is said to be linearly degenerate if its eigenvalues (assumed real and distinct) are constant in the direction of the corresponding eigenvectors. Explicitly, $L_{r^{i}} \lambda^{i}=0$, no summation, where $L_{r^{i}}$ denotes Lie derivative in the direction of the eigenvector $r^{i}$, and $A r^{i}=\lambda^{i} r^{i}$. For quasilinear systems, the property of linear degeneracy is known to be related to the impossibility of breakdown of smooth initial data [25]. In terms of the original functions $f\left(u_{1}, v_{1}\right)$ and $h\left(u_{1}, v_{1}\right)$, the conditions of linear degeneracy reduce to a pair of second-order differential constraints [7],

$$
\begin{aligned}
& \left(f_{u_{1}}-h_{v_{1}}\right) f_{u_{1} u_{1}}+2 h_{u_{1}} f_{u_{1} v_{1}}+h_{u_{1}} h_{v_{1} v_{1}}+f_{v_{1}} h_{u_{1} u_{1}}=0, \\
& \left(h_{v_{1}}-f_{u_{1}}\right) h_{v_{1} v_{1}}+2 f_{v_{1}} h_{u_{1} v_{1}}+f_{v_{1}} f_{u_{1} u_{1}}+h_{u_{1}} f_{v_{1} v_{1}}=0 .
\end{aligned}
$$

Requiring that all traveling wave reductions of a multi-dimensional system to $2 \mathrm{D}$ are linearly degenerate in the above sense, we obtain differential characterisation of linear degeneracy:

Proposition 1 [7]. System (9) is linearly degenerate if and only if the functions $f$ and $h$ satisfy the relations

$$
\begin{aligned}
& \operatorname{Sym}_{\{i, j, k\}}\left(\left(f_{u_{k}}-h_{v_{k}}\right) f_{u_{i} u_{j}}+h_{u_{k}}\left(f_{u_{i} v_{j}}+f_{u_{j} v_{i}}\right)+f_{v_{k}} h_{u_{i} u_{j}}+h_{u_{k}} h_{v_{i} v_{j}}\right)=0, \\
& \operatorname{Sym}_{\{i, j, k\}}\left(\left(h_{v_{k}}-f_{u_{k}}\right) h_{v_{i} v_{j}}+f_{v_{k}}\left(h_{u_{i} v_{j}}+h_{u_{j} v_{i}}\right)+h_{u_{k}} f_{v_{i} v_{j}}+f_{v_{k}} f_{u_{i} u_{j}}\right)=0,
\end{aligned}
$$

where Sym denotes complete symmetrisation over $i, j, k \in\{1,2,3\}$. Note that conditions (10) are invariant under the equivalence group $\mathbf{S L}(6)$.

The key observation is that second-order overdetermined system (10) is not in involution: its differential prolongation results in the two branches characterised by additional second-order differential constraints. The first branch leads to Monge-Ampère systems (10 additional secondorder constraints). The second branch corresponds to general linearly degenerate systems (4 additional second-order constraints), see Section 3.2 for the details of this analysis.

\subsection{Summary of the main results}

Our results imply that several seemingly different approaches to integrability described above lead to one and the same class of systems (1).

Theorem 1 Under the non-degeneracy assumption, the following conditions are equivalent:

(a) System (1) is integrable by the method of hydrodynamic reductions.

(b) Conformal structure $[g]$ defined by the characteristic variety of system (1) is self-dual on every solution.

(c) System (1) is linearly degenerate.

(d) The associated sixfold $X \subset \mathbf{G r}(4,6)$ is either a codimension two linear section of the Plücker embedding $\mathbf{G r}(4,6) \hookrightarrow \mathbb{P}^{14}$, or the image of a quadratic map $\mathbb{P}^{6} \rightarrow \mathbf{G r}(4,6)$. 
Theorem 1 and the results of [3] imply that any integrable system (1) possesses a Lax representation in parameter-dependent commuting vector fields. Integral surfaces of these vector fields give rise to $\alpha$-surfaces of the conformal structure $[g]$.

Examples of integrable systems (1) are discussed in Section 2. The proof of Theorem 1 is given in Section 3. All calculations are based on computer algebra systems Mathematica and Maple (these only utilise symbolic polynomial algebra over $\mathbb{Q}$, so the results are rigorous). The programmes are available from the arXiv supplement to this paper.

\section{Examples and classification results}

In this section we discuss examples of $4 \mathrm{D}$ systems which, as will be demonstrated in Section 3, exhaust the list of all integrable systems of type (1).

\subsection{Monge-Ampère systems}

Systems of Monge-Ampère type correspond to sixfolds $X \subset \mathbf{G r}(4,6)$ that can be obtained as codimension two linear sections of the Plücker embedding of the Grassmannian. Recall that $\operatorname{Gr}(4,6)$ is an 8-dimensional algebraic variety of degree 14 embedded into $\mathbb{P}^{14}$. All 2-component systems of Monge-Ampère type are integrable. They were classified in our recent paper [8].

Proposition 2 [8]. In four dimensions, any non-degenerate system of Monge-Ampère type is SL(6)-equivalent to one of the following normal forms:

1. $u_{2}-v_{1}=0, \quad u_{3}+v_{4}=0$

2. $u_{2}-v_{1}=0, \quad u_{3}+v_{4}+u_{1} v_{2}-u_{2} v_{1}=0$,

3. $u_{2}-v_{1}=0, \quad u_{3} v_{4}-u_{4} v_{3}-1=0$,

4. $u_{2}-v_{1}=0, \quad u_{1}+v_{2}+u_{3} v_{4}-u_{4} v_{3}=0$.

All these systems can be reduced to various heavenly-type equations. Introducing the potential $w$ such that $w_{1}=u, w_{2}=v$ one obtains the linear ultrahyperbolic equation $w_{13}+w_{24}=0$, the second heavenly equation $w_{13}+w_{24}+w_{11} w_{22}-w_{12}^{2}=0$ [24], the first heavenly equation $w_{13} w_{24}-w_{14} w_{23}-1=0$ [24], and the Husain equation $w_{11}+w_{22}+w_{13} w_{24}-w_{14} w_{23}=0$ [18], respectively. All of them originate from self-dual Ricci-flat geometry. Their integrability by the method of hydrodynamic reductions was established in [12, 13].

Representing system (1) in evolutionary form (9) one obtains a differential characterisation of the Monge-Ampère property.

Proposition 3 [8]. The necessary and sufficient conditions for system (9) to be of MongeAmpère type are equivalent to the following second-order relations for $f$ and $h$,

$$
\begin{gathered}
f_{u_{i} u_{i}}=\frac{2 h_{u_{i}}}{h_{v_{i}}-f_{u_{i}}} f_{u_{i} v_{i}}, \quad f_{v_{i} v_{i}}=\frac{2 f_{v_{i}}}{f_{u_{i}}-h_{v_{i}}} f_{u_{i} v_{i}}, \\
f_{u_{i} u_{j}}=\frac{h_{u_{j}}}{h_{v_{i}}-f_{u_{i}}} f_{u_{i} v_{i}}+\frac{h_{u_{i}}}{h_{v_{j}}-f_{u_{j}}} f_{u_{j} v_{j}}, \quad f_{v_{i} v_{j}}=\frac{f_{v_{j}}}{f_{u_{i}}-h_{v_{i}}} f_{u_{i} v_{i}}+\frac{f_{v_{i}}}{f_{u_{j}}-h_{v_{j}}} f_{u_{j} v_{j}}, \\
f_{u_{i} v_{j}}+f_{u_{j} v_{i}}=\frac{f_{u_{j}}-h_{v_{j}}}{f_{u_{i}}-h_{v_{i}}} f_{u_{i} v_{i}}+\frac{f_{u_{i}}-h_{v_{i}}}{f_{u_{j}}-h_{v_{j}}} f_{u_{j} v_{j}},
\end{gathered}
$$


where $i, j=1,2,3$. Equations for $h$ can be obtained by the simultaneous substitution $f \leftrightarrow h$ and $u \leftrightarrow v$ (30 second-order relations altogether).

Table 1 below contains the (Lie algebra) structure of the stabilisers of Monge-Ampère systems under the action of the equivalence group SL(6) (note that different cases are distinguished by the dimensions of the stabilisers).

Table 1: types of isotropy algebras $\mathfrak{s} \subset \mathfrak{s l}_{6}$ of Monge-Ampère systems in $4 \mathrm{D}$

\begin{tabular}{|c|c|c|}
\hline System of equations & $\operatorname{dim}(\mathfrak{s})$ & Levi decomposition of the algebra $\mathfrak{s}$ \\
\hline $\begin{array}{l}\text { 1: linear ultrahyperbolic } \\
u_{2}-v_{1}=0 \\
u_{3}+v_{4}=0\end{array}$ & 13 & $\begin{array}{c}\mathfrak{s}=\mathfrak{s}_{0} \oplus \mathfrak{s}_{1} \text { graded by } r \in \mathfrak{z}\left(\mathfrak{g l}_{2}\right) \\
\mathfrak{s}=\left(\mathfrak{s l}_{2} \oplus \mathfrak{g l}_{2}\right) \ltimes\left(\mathbb{R}^{2} \otimes \mathbb{R}^{3}\right) \\
\mathfrak{s} \text { is self-normalizing }\end{array}$ \\
\hline $\begin{array}{l}\text { 2: 2nd heavenly } \\
u_{2}-v_{1}=0 \\
u_{3}+v_{4}+u_{1} v_{2}-u_{2} v_{1}=0\end{array}$ & 11 & $\begin{array}{c}\mathfrak{s}=\mathfrak{s}_{0} \oplus \mathfrak{s}_{1} \oplus \mathfrak{s}_{2} \text { graded by } r \in \mathfrak{z}\left(\mathfrak{g l}_{2}\right) \\
\mathfrak{s}=\mathfrak{g l}_{2} \ltimes\left(\left(\mathbb{R}^{1}+\mathbb{R}^{3}\right) \ltimes \mathbb{R}^{3}\right) \\
\mathfrak{s} \text { is self-normalizing }\end{array}$ \\
\hline $\begin{array}{l}3: 1 \text { st heavenly } \\
u_{2}-v_{1}=0 \\
u_{3} v_{4}-u_{4} v_{3}-1=0\end{array}$ & 10 & $\begin{array}{c}\mathfrak{s}=\mathfrak{s}_{0} \oplus \mathfrak{s}_{1} \text { graded by } r \in \mathfrak{z}\left(\mathfrak{g l}_{2}\right) \\
\mathfrak{s}=\mathfrak{s l}_{2} \oplus\left(\mathfrak{g l}_{2} \ltimes \mathbb{R}^{3}\right) \\
\mathfrak{s} \text { is not self-normalizing }\end{array}$ \\
\hline $\begin{array}{l}\text { 4: Husain system } \\
u_{2}-v_{1}=0 \\
u_{1}+v_{2}+u_{3} v_{4}-u_{4} v_{3}=0\end{array}$ & 9 & $\begin{array}{c}\text { semi-simple } \\
\mathfrak{s}=\mathfrak{s l}_{2} \oplus \mathfrak{s l}(2, \mathbb{C})_{\mathbb{R}} \\
\mathfrak{s} \text { is not self-normalizing }\end{array}$ \\
\hline
\end{tabular}

\section{Notes:}

(1) The factors $\mathbb{R}^{2}, \mathbb{R}^{3}$ are irreducible representations of the corresponding $\mathfrak{s l}_{2}$ (same for the $\mathfrak{s l}_{2}$ factor in $\mathfrak{g l}_{2}=\mathfrak{s l}_{2} \oplus \mathbb{R}$ ) in cases $1-3$.

(2) Lie algebra structure of the nilradical $\mathbb{R}^{1}+\mathbb{R}_{a}^{3}+\mathbb{R}_{b}^{3}$ of $\mathfrak{s}$ in case $2:\left[\mathbb{R}^{1}, \mathbb{R}_{a}^{3}\right]=\mathbb{R}_{b}^{3},\left[\mathbb{R}_{a}^{3}, \mathbb{R}_{a}^{3}\right]=\mathbb{R}_{b}^{3}$ $\left(\mathfrak{s l}_{2}\right.$-equivariance fixes the brackets uniquely).

(3) We indicate real forms of the equations in the left-hand side. Since the classification is over $\mathbb{C}$, the corresponding complex forms should be taken, e.g. $\left(\mathfrak{s l}_{2}^{\mathbb{C}}\right)^{\oplus 3}$ in case 4 .

(4) Normalizers of $\mathfrak{s} \subset \mathfrak{s l}_{6}$ in cases 3, 4 both have dimensions 11 (extension of the $\mathfrak{s l}_{2}$ factor to $\mathfrak{g l}_{2}$ in case 3 and of $\mathfrak{s}$ to the trace-free part of $\mathfrak{g l}_{2} \oplus \mathfrak{g l}(2, \mathbb{C})_{\mathbb{R}}$ in case 4$)$.

\section{$2.2 \quad$ Linearisable systems}

In this section we characterise systems (1) which can be linearised by a transformation from the equivalence group SL(6). Note that linearisable systems are necessarily of Monge-Ampère type.

Theorem 4. Under the non-degeneracy assumption, the following conditions are equivalent:

(a) System (1) is linearisable by a transformation from the equivalence group $\mathbf{S L}(6)$.

(b) System (1) is invariant under a 13-dimensional subgroup of $\mathbf{S L}(6)$.

(c) The characteristic variety of system (1) defines a conformal structure $[g]$ which is flat on every solution: $W=0$.

Proof. Equivalence $(a) \Longleftrightarrow(b)$ : Consider a non-degenerate linear system, say $u_{2}-v_{1}=$ $0, u_{3}+v_{4}=0$ (note that all non-degenerate linear systems of type (1) are $\mathbf{S L}$ (6)-equivalent). This system is invariant under a 13-dimensional subgroup of SL(6) with the following infinitesimal 
generators (we use the notations of Section 1.4):

$$
\begin{gathered}
\mathbf{U}_{1}, \quad \mathbf{U}_{4}, \quad \mathbf{V}_{2}, \quad \mathbf{V}_{3}, \quad \mathbf{U}_{2}+\mathbf{V}_{1}, \quad \mathbf{U}_{3}-\mathbf{V}_{4} \\
\mathbf{X}_{11}+\mathbf{X}_{22}, \quad \mathbf{X}_{33}+\mathbf{X}_{44}, \quad \mathbf{X}_{14}-\mathbf{X}_{23}, \quad \mathbf{X}_{41}-\mathbf{X}_{32}, \\
\mathbf{X}_{12}-\mathbf{X}_{43}+\mathbf{L}_{12}, \quad \mathbf{X}_{21}-\mathbf{X}_{34}+\mathbf{L}_{21}, \quad \mathbf{X}_{22}+\mathbf{X}_{33}+\mathbf{L}_{22}
\end{gathered}
$$

This Lie algebra is isomorphic to the semi-direct product $\left(V_{1} \otimes V_{2}\right) \rtimes\left(\mathfrak{g l}_{2} \times \mathfrak{s} \mathfrak{l}_{2}\right)$, where $V_{1} \otimes V_{2} \simeq \mathbb{R}^{6}$ is the tensor product of the standard representation $V_{1}$ of $\mathfrak{g l}_{2}=\mathfrak{s l}_{2} \oplus \mathbb{R}$, and the representation $V_{2}$ of $\mathfrak{s l}_{2}$. Here $\mathfrak{g l}_{2}$ (resp. $\mathfrak{s l}_{2}$ ) acts on the first (resp. second) factor of $V_{1} \otimes V_{2}$.

To establish the converse, let $G$ be the symmetry group of system (1). We can always assume that the point $o$, specified by $u_{i}=v_{i}=0$, belongs to the sixfold $X \subset \mathbf{G r}(4,6)$ corresponding to our system. Let $G_{O}$ be the stabiliser of this point in $G$. Note that $\operatorname{dim} G-\operatorname{dim} G_{o} \leq 6$, as $G$ takes $X$ to itself. The stabiliser $P$ of the point $o$ is spanned by infinitesimal generators $\mathbf{X}_{i j}, \mathbf{L}_{i j}, \mathbf{P}_{i}, \mathbf{Q}_{i}$. Since the system is non-degenerate, we can bring it to a canonical form

$$
u_{2}=v_{1}+o\left(u_{i}, v_{i}\right), \quad u_{3}=-v_{4}+o\left(u_{i}, v_{i}\right) .
$$

This form (together with the point $o$ ) is stabilised by 7 elements of $P$ listed in the last two lines of (12). Thus, $\operatorname{dim} G_{O} \leq 7$ so that $\operatorname{dim} G \leq 13$. The equality holds only if $\operatorname{dim} G_{O}=7$. However, the generator $\mathbf{X}_{11}+\mathbf{X}_{22}+\mathbf{X}_{33}+\mathbf{X}_{44}$ acts by non-trivial rescalings on terms of order 2 and higher in (13). Hence, for $\operatorname{dim} G_{o}=7$, all higher-order terms must vanish identically, leading to a linear system.

Equivalence $(a) \Longleftrightarrow(c)$ : Let us represent system (1) in evolutionary form (9) and take the corresponding conformal structure $[g]$. Conformal flatness is equivalent to the vanishing of the Weyl tensor

$$
W_{i j k l}=R_{i j k l}-w_{i k} g_{j l}-w_{j l} g_{i k}+w_{j k} g_{i l}+w_{i l} g_{j k}=0,
$$

where $R_{i j k l}=g_{i s} R_{j k l}^{s}$ is the curvature tensor, $w_{i j}=\frac{1}{2} R_{i j}-\frac{R}{12} g_{i j}$ is the Schouten tensor, $R_{i j}$ is the Ricci tensor, and $R$ is the scalar curvature. Calculating (14) and using equations (9) along with their differential consequences to eliminate all higher-order partial derivatives of $u$ and $v$ containing differentiation by $x^{4}$, we obtain expressions that have to vanish identically in the remaining higher-order derivatives (no more than third-order derivatives are involved in this calculation). In particular, equating to zero coefficients at the remaining third-order derivatives of $u$ and $v$ we obtain 34 second-order relations for $f$ and $h$ that contain 30 relations (11) governing Monge-Ampère systems, plus 4 extra (more complicated) relations. The easiest way to finish the proof is to note that according to Proposition 2 of Section 2.1, any 4D system of MongeAmpère type is SL(6)-equivalent to one of the four normal forms, and direct verification shows that conformal structures defined by characteristic varieties of the last three (non-linearisable) normal forms are not flat on generic solutions. Thus, the above 34 second-order relations are nothing but the linearisability conditions. This finishes the proof of Proposition 4.

\subsection{Systems associated with quadratic maps $\mathbb{P}^{6} \rightarrow \operatorname{Gr}(4,6)$}

In this section we classify integrable systems (1) which correspond to sixfolds $X \subset \mathbf{G r}(4,6)$ resulting as images of quadratic maps $\mathbb{P}^{6} \rightarrow \mathbf{G r}(4,6)$. These maps come from the following geometric construction.

Consider two vector spaces $V$ and $W$. Let $A \in \operatorname{Hom}(W, V)$ and $B \in \operatorname{Hom}(W, V)$ be two linear maps. The collection of 2-planes $A x \wedge B x, x \in W$, defines a subvariety of $\operatorname{Gr}(2, V)$, the image 
of a quadratic map $\mathbb{P}(W) \rightarrow \mathbf{G r}(2, V)$. In the particular case $V=W$ this construction goes back to Chasles [4] who considered the locus of lines spanned by an argument and the value of a projective transformation; see also [5], p. 556. Quadratic maps $\mathbb{P}^{6} \rightarrow \mathbf{G r}(2,6)$ result from the above construction when $\operatorname{dim} V=6, \operatorname{dim} W=7$. This gives a map $\mathbb{P}(W)=\mathbb{P}^{6} \rightarrow \mathbf{G r}(2, V)$, leading by duality to a quadratic map $\mathbb{P}^{6} \rightarrow \mathbf{G r}\left(4, V^{*}\right)=\mathbf{G r}(4,6)$.

In coordinates, this reads as follows. Consider projective space $\mathbb{P}(W)=\mathbb{P}^{6}$ with homogeneous coordinates $\xi=\left(\xi^{1}: \xi^{2}: \xi^{3}: \xi^{4}: \xi^{5}: \xi^{6}: \xi^{7}\right)$. Let $A$ and $B$ be two $7 \times 6$ matrices representing the corresponding linear maps. Introduce the $2 \times 6$ matrix of linear forms on $W$,

$$
\left(\begin{array}{llllll}
\eta^{1} & \eta^{2} & \eta^{3} & \eta^{4} & \eta^{5} & \eta^{6} \\
\tau^{1} & \tau^{2} & \tau^{3} & \tau^{4} & \tau^{5} & \tau^{6}
\end{array}\right)
$$

where $\eta=\xi A$ and $\tau=\xi B$. The Plücker coordinates $p^{i j}=\eta^{i} \tau^{j}-\eta^{j} \tau^{i}$ define a quadratic map $\mathbb{P}^{6} \rightarrow \mathbf{G r}(2,6) \subset \mathbb{P}^{14}$. By duality, this gives a sixfold $X \subset \mathbf{G r}(4,6)$, and the corresponding system (1). Explicit parametric formulae can be obtained from the factorised representation,

$$
\left(\begin{array}{llllll}
\eta^{1} & \eta^{2} & \eta^{3} & \eta^{4} & \eta^{5} & \eta^{6} \\
\tau^{1} & \tau^{2} & \tau^{3} & \tau^{4} & \tau^{5} & \tau^{6}
\end{array}\right)=\left(\begin{array}{ll}
\eta^{5} & \eta^{6} \\
\tau^{5} & \tau^{6}
\end{array}\right)\left(\begin{array}{llllll}
u_{1} & u_{2} & u_{3} & u_{4} & 1 & 0 \\
v_{1} & v_{2} & v_{3} & v_{4} & 0 & 1
\end{array}\right)
$$

which gives $u_{i}=p^{i 6} / p^{56}, v_{i}=p^{i 5} / p^{65}, i=1, \ldots, 4$. Eliminating $\xi$ 's, we obtain two relations among $u_{i}, v_{i}$, which constitute the required system $\Sigma(X)$.

Tables $2-6$ below comprise a complete list of resulting systems (1) labelled by JordanKronecker normal forms [16] of the matrix pencil $A, B$ (see the end of this section for an illustrative calculation leading to the first case of Table 2). Note that $A$ and $B$ are defined up to transformations $A \rightarrow P A Q, B \rightarrow P B Q$, where the $7 \times 7$ matrix $P$ is responsible for a change of basis in $W$ and the $6 \times 6$ matrix $Q$ corresponds to the action of the equivalence group $\mathbf{S L}(6)$. Modulo these transformations, $A$ and $B$ must have exactly one Kronecker block of the size $(n+1) \times n$, for $n=2, \ldots, 6$ (the cases of a single $2 \times 1$ Kronecker block, as well as of more than one Kronecker blocks, lead to either degenerate or linear systems). We group systems according to the size of the Kronecker block. Within each table, systems are labelled by Serge types of the remaining Jordan block. In all cases (with the exception of the most generic system from Table 6 ) we have chosen canonical forms which, via elimination of $u$, imply second-order equations for $v$. We also present the associated dispersionless Lax pairs in the form of two commuting $\lambda$-dependent vector fields, $[X, Y]=0$.

Table 2: canonical forms with one $3 \times 2$ Kronecker block

\begin{tabular}{|l|l|l|l|}
\hline Segre type & Canonical form & Equation for $v$ & Lax pair \\
\hline$[111]]$ & $\alpha u_{2} v_{1}=u_{1} v_{2}$ & $\left(\frac{v_{2}}{v_{1}}\right)_{4}-\left(\frac{v_{2}}{v_{1}}\right)_{3}=(\alpha-1)\left(\frac{v_{3}}{v_{1}}\right)_{2}$ & $\begin{array}{l}X=\partial_{1}+\frac{\lambda-\alpha}{1-\lambda} \frac{v_{1}}{v_{2}} \partial_{2} \\
\end{array}$ \\
& $u_{4} v_{1}=u_{1} v_{3}$ & & $Y=\partial_{4}-\lambda \partial_{3}+(\lambda-\alpha) \frac{v_{3}}{v_{2}} \partial_{2}$ \\
\hline$[211]$ & $u_{2} v_{1}-u_{1} v_{2}=v_{1} v_{2}$ & $\left(\frac{v_{2}}{v_{1}}\right)_{3}=\left(\frac{v_{4}}{v_{1}}\right)_{2}$ & $X=\partial_{1}+(\lambda-1) \frac{v_{1}}{v_{2}} \partial_{2}$ \\
& $u_{4} v_{1}-u_{1} v_{4}=v_{1} v_{3}$ & & $Y=\partial_{4}-\lambda \partial_{3}+(\lambda-1) \frac{v_{4}}{v_{2}} \partial_{2}$ \\
\hline$[22]$ & $u_{2} v_{1}-u_{1} v_{2}=v_{1}^{2}$ & $\left(\frac{v_{2}}{v_{1}}\right)_{3}=\left(\frac{v_{4}}{v_{1}}\right)_{1}$ & $X=\partial_{2}-\left(\lambda+\frac{v_{2}}{v_{1}}\right) \partial_{1}$ \\
& $u_{4} v_{1}-u_{1} v_{4}=v_{1} v_{3}$ & & $Y=\partial_{4}-\lambda \partial_{3}-\frac{v_{4}}{v_{1}} \partial_{1}$ \\
\hline$[31]$ & $u_{2}=-v_{1} v_{2}$ & $v_{23}+v_{2} v_{14}-v_{4} v_{12}=0$ & $X=\partial_{2}+\lambda v_{2} \partial_{1}$ \\
& $u_{4}=v_{3}-v_{1} v_{4}$ & & $Y=\partial_{4}-\lambda \partial_{3}+\lambda v_{4} \partial_{1}$ \\
\hline$[4]$ & $u_{1}=v_{2}-v_{1}^{2}$ & $v_{24}-v_{13}+v_{4} v_{11}-v_{1} v_{14}=0$ & $X=\partial_{2}-\left(v_{1}+\lambda\right) \partial_{1}$ \\
& $u_{4}=v_{3}-v_{1} v_{4}$ & & $Y=\partial_{3}-v_{4} \partial_{1}-\lambda \partial_{4}$ \\
\hline
\end{tabular}


Table 3: canonical forms with one $4 \times 3$ Kronecker block

\begin{tabular}{|c|c|c|c|}
\hline Segre type & Canonical form & Equation for $v$ & Lax pair \\
\hline$[111]$ & $\begin{array}{l}u_{3} v_{1}=\alpha\left(v_{2}-v_{3}\right) u_{1} \\
u_{4} v_{1}=\alpha\left(v_{3}-v_{4}\right) u_{1}\end{array}$ & $\begin{array}{l}m_{4}+\alpha m n_{1}=n_{3}+\alpha n m_{1} \\
m=\frac{v_{2}-v_{3}}{v_{1}}, n=\frac{v_{3}-v_{4}}{v_{1}}\end{array}$ & $\begin{array}{c}X=\partial_{2}-c(m+\lambda n) \partial_{1}-\lambda^{2} \partial_{4} \\
Y=\partial_{3}-c n \partial_{1}-\lambda \partial_{4} \\
\quad c=1+\alpha-\lambda \alpha\end{array}$ \\
\hline$[21]$ & $\begin{array}{l}u_{3} v_{1}-u_{1} v_{3}=\left(v_{2}-\alpha v_{3}\right) v_{1} \\
u_{4} v_{1}-u_{1} v_{4}=\left(v_{3}-\alpha v_{4}\right) v_{1}\end{array}$ & $\begin{aligned} &\left(\partial_{2}-\alpha \partial_{3}\right) \frac{v_{4}}{v_{1}} \\
&=\left(\partial_{3}-\alpha \partial_{4}\right) \frac{v_{3}}{v_{1}}\end{aligned}$ & $\begin{array}{l}X=\partial_{2}+(\lambda-\alpha) \frac{\lambda v_{4}+v_{3}}{v_{1}} \partial_{1}-\lambda^{2} \partial_{4} \\
Y=\partial_{3}+(\lambda-\alpha) \frac{v_{4}}{v_{1}} \partial_{1}-\lambda \partial_{4}\end{array}$ \\
\hline$[3]$ & $\begin{array}{l}u_{3}=v_{2}-v_{1} v_{3} \\
u_{4}=v_{3}-v_{1} v_{4}\end{array}$ & $v_{24}-v_{33}=v_{3} v_{14}-v_{4} v_{13}$ & $\begin{array}{l}X=\partial_{2}-\left(\lambda v_{4}+v_{3}\right) \partial_{1}-\lambda^{2} \partial_{4} \\
Y=\partial_{3}-v_{4} \partial_{1}-\lambda \partial_{4}\end{array}$ \\
\hline
\end{tabular}

Table 4: canonical forms with one $5 \times 4$ Kronecker block

\begin{tabular}{|l|l|l|l|}
\hline Segre type & Canonical form & Equation for $v$ & Lax pair \\
\hline$[11]$ & $u_{3}\left(v_{2}-v_{1}\right)=u_{2}\left(v_{3}-v_{2}\right)$ & $m_{3}+m n_{1}=n_{2}+n m_{1}$ & $X=\partial_{3}-(\lambda+m) \partial_{2}+\lambda m \partial_{1}$ \\
& $u_{4}\left(v_{2}-v_{1}\right)=u_{2}\left(v_{4}-v_{3}\right)$ & $m=\frac{v_{3}-v_{2}}{v_{2}-v_{1}}, n=\frac{v_{4}-v_{3}}{v_{2}-v_{1}}$ & $Y=\partial_{4}-\left(\lambda^{2}+\lambda m+n\right) \partial_{2}+\left(\lambda^{2} m+\lambda n\right) \partial_{1}$ \\
\hline$[2]$ & $v_{3}\left(u_{2}-v_{1}\right)=v_{2}\left(u_{3}-v_{2}\right)$ & $m_{3}+m n_{1}=n_{2}+n m_{1}$ & $X=\partial_{3}-(\lambda+m) \partial_{2}+\lambda m \partial_{1}$ \\
& $v_{4}\left(u_{2}-v_{1}\right)=v_{2}\left(u_{4}-v_{3}\right)$ & $m=\frac{v_{3}}{v_{2}}, n=\frac{v_{4}}{v_{2}}$ & $Y=\partial_{4}-\left(\lambda^{2}+\lambda m+n\right) \partial_{2}+\left(\lambda^{2} m+\lambda n\right) \partial_{1}$ \\
\hline
\end{tabular}

Table 5: canonical form with one $6 \times 5$ Kronecker block

\begin{tabular}{|l|l|l|}
\hline Segre type & Canonical form & Equation for $v$ and Lax pair \\
\hline$[1]$ & $\frac{u_{2}-u_{1} v_{1}}{v_{2}-v_{1}^{2}}=\frac{u_{3}-u_{1} v_{2}}{v_{3}-v_{1} v_{2}}=\frac{u_{4}-u_{1} v_{3}}{v_{4}-v_{1} v_{3}}$ & $m_{3}+m n_{1}=n_{2}+n m_{1}$ \\
& & $X=\partial_{3}-(\lambda+m) \partial_{2}+(\lambda m-a) \partial_{1}$ \\
& & $Y=\partial_{4}-\left(\lambda^{2}+\lambda m+n\right) \partial_{2}+\left(\lambda^{2} m+\lambda n-\lambda a-b\right) \partial_{1}$ \\
& & $m=\frac{v_{3}-v_{1} v_{2}}{v_{2}-v_{1}^{2}}, n=\frac{v_{4}-v_{1} v_{3}}{v_{2}-v_{1}^{2}}, a=\frac{v_{2}^{2}-v_{1} v_{3}}{v_{2}-v_{1}^{2}}, b=\frac{v_{2} v_{3}-v_{1} v_{4}}{v_{2}-v_{1}^{2}}$ \\
\hline
\end{tabular}

Table 6: canonical form with one $7 \times 6$ Kronecker block

\begin{tabular}{|l|l|l|}
\hline Segre type & Canonical form & Lax pair \\
\hline$[0]$ & $\frac{u_{2}-u_{1} v_{1}}{v_{2}-u_{1}-v_{1}^{2}}=\frac{u_{3}-u_{1} v_{2}}{v_{3}-u_{2}-v_{1} v_{2}}$ & note that there is no equation for $v$ in this case \\
& $=\frac{u_{4}-u_{1} v_{3}}{v_{4}-u_{3}-v_{1} v_{3}}$ & $X=\partial_{3}-(\lambda+m) \partial_{2}+(\lambda m-a) \partial_{1}$ \\
& & $Y=\partial_{4}-\left(\lambda^{2}+\lambda m+n\right) \partial_{2}+\left(\lambda^{2} m+\lambda n-\lambda a-b\right) \partial_{1}$ \\
& & $m=\frac{u_{3}-u_{1} v_{2}}{u_{2}-u_{1} v_{1}}, n=\frac{u_{4}-u_{1} v_{3}}{u_{2}-u_{1} v_{1}}, a=\frac{u_{2} v_{2}-u_{3} v_{1}}{u_{2}-u_{1} v_{1}}, b=\frac{u_{2} v_{3}-u_{4} v_{1}}{u_{2}-u_{1} v_{1}}$ \\
\hline
\end{tabular}

Remark. Note that both systems from Table 4 are related to (one and the same!) quasilinear system for the corresponding variables $m, n$, namely

$$
m_{4}-n_{3}+m n_{2}-n m_{2}=0, \quad m_{3}-n_{2}+m n_{1}-n m_{1}=0
$$

(indeed, in terms of these variables their Lax pairs are identically the same). Thus, although the original systems are not equivalent under the natural equivalence group $\mathbf{S L}(6)$, the corresponding equations for $v$ are related by a Bäcklund transformation. System (15) can be viewed as a travelling wave reduction of the $6 \mathrm{D}$ integrable system

$$
m_{6}-n_{5}+m n_{4}-n m_{4}=0, \quad m_{3}-n_{2}+m n_{1}-n m_{1}=0
$$


discussed in [13].

Similarly, the coincidence of Lax pairs from Tables 5 and 6 indicates that the corresponding systems can be considered as (nonlinear) reductions of one and the same first-order 4-component system for the variables $a, b, m, n$ resulting from the commutativity condition $[X, Y]=0$. This 4-component system can be viewed as yet another equivalent form of the equations governing hyper-Hermitian conformal structures in 4D [10].

Notice that the absence of terms with $\partial_{\lambda}$ in the Lax representations from Tables 2-6 means that all solutions of the above systems carry hyper-Hermitian geometry [9], which is associated to the canonical conformal structure.

Example. Let us give details of calculations in the case when the pair $A, B$ contains one $3 \times 2$ Kronecker block (upper left) and a $4 \times 4$ Jordan block of Segre type [1111], explicitly,

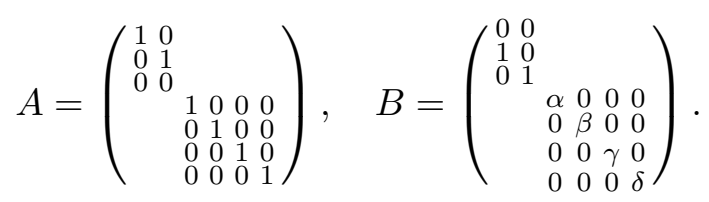

The corresponding $2 \times 6$ matrix of linear forms is

$$
\left(\begin{array}{cccccc}
\xi^{1} & \xi^{2} & \xi^{4} & \xi^{5} & \xi^{6} & \xi^{7} \\
\xi^{2} & \xi^{3} & \alpha \xi^{4} & \beta \xi^{5} & \gamma \xi^{6} & \delta \xi^{7}
\end{array}\right)=\left(\begin{array}{cc}
\xi^{6} & \xi^{7} \\
\gamma \xi^{6} & \delta \xi^{7}
\end{array}\right)\left(\begin{array}{cccccc}
u_{1} & u_{2} & u_{3} & u_{4} & 1 & 0 \\
v_{1} & v_{2} & v_{3} & v_{4} & 0 & 1
\end{array}\right)
$$

so that

$$
\begin{aligned}
& u_{1}=\frac{\delta \xi^{1}-\xi^{2}}{(\delta-\gamma) \xi^{6}}, \quad u_{2}=\frac{\delta \xi^{2}-\xi^{3}}{(\delta-\gamma) \xi^{6}}, \quad u_{3}=\frac{(\delta-\alpha) \xi^{4}}{(\delta-\gamma) \xi^{6}}, \quad u_{4}=\frac{(\delta-\beta) \xi^{5}}{(\delta-\gamma) \xi^{6}} \\
& v_{1}=\frac{\gamma \xi^{1}-\xi^{2}}{(\gamma-\delta) \xi^{7}}, \quad v_{2}=\frac{\gamma \xi^{2}-\xi^{3}}{(\gamma-\delta) \xi^{7}}, \quad v_{3}=\frac{(\gamma-\alpha) \xi^{4}}{(\gamma-\delta) \xi^{7}}, \quad v_{4}=\frac{(\gamma-\beta) \xi^{5}}{(\gamma-\delta) \xi^{7}}
\end{aligned}
$$

The elimination of $\xi$ 's leads to the following relations:

$$
u_{3} v_{4}=\frac{(\delta-\beta)(\gamma-\alpha)}{(\delta-\alpha)(\gamma-\beta)} u_{4} v_{3}, \quad u_{4}\left(v_{2}-\delta v_{1}\right)=\frac{\delta-\beta}{\gamma-\beta} v_{4}\left(u_{2}-\gamma u_{1}\right) .
$$

Modulo equivalence transformations, this system is reducible to the first case of Table 2 .

\subsection{Symmetries of general linearly degenerate systems}

The equivalence group SL(6) preserves both the class of Monge-Ampère equations and the class given by the Chasles construction. The stabilizer of an equation is its linear symmetry group (the full group of point symmetries of an integrable system is normally infinite-dimensional).

For Monge-Ampère systems, the Lie algebras $\mathfrak{s}$ corresponding to these groups were indicated in Table 1. Below we provide some data on the isotropy algebras for general linearly degenerate systems from Tables 2-6. We denote by $\mathfrak{c}(\mathfrak{s})=\left\{g \in \mathfrak{s l}_{6}:[g, \mathfrak{s}]=0\right\}$ the centralizer of $\mathfrak{s}$, and by $\mathfrak{n}(\mathfrak{s})=\left\{g \in \mathfrak{s l}_{6}:[g, \mathfrak{s}] \subset \mathfrak{s}\right\}$ the normalizer of $\mathfrak{s}$. 
Table 7: types of isotropy algebras $\mathfrak{s} \subset \mathfrak{s l}_{6}$ for general linearly degenerate systems in $4 \mathrm{D}$

\begin{tabular}{|l|c|c|c|c|l|}
\hline Segre type & $\operatorname{dim} \mathfrak{s}$ & $\operatorname{dim} \mathfrak{c}(\mathfrak{s})$ & $\operatorname{dim} \mathfrak{n}(\mathfrak{s})$ & Lie algebra type & dim. derived ser. \\
\hline$[111]$ & 8 & 0 & 8 & solvable & $(8,4,0)$ \\
\hline$[211]$ & 8 & 0 & 8 & solvable & $(8,4,0)$ \\
\hline$[22]$ & 9 & 0 & 9 & solvable & $(9,6,2,0)$ \\
\hline$[31]$ & 9 & 0 & 9 & solvable & $(9,6,2,0)$ \\
\hline$[4]$ & 10 & 0 & 10 & solvable & $(10,8,5,1,0)$ \\
\hline$[111]$ & 6 & 2 & 8 & solvable & $(6,3,0)$ \\
\hline$[21]$ & 7 & 1 & 8 & solvable & $(7,4,1,0)$ \\
\hline$[3]$ & 8 & 0 & 8 & solvable & $(8,6,3,0)$ \\
\hline$[11]$ & 5 & 3 & 7 & solvable & $(5,2,0)$ \\
\hline$[2]$ & 6 & 0 & 6 & solvable & $(6,4,1,0)$ \\
\hline$[1]$ & 4 & 0 & 4 & solvable & $(4,2,0)$ \\
\hline$[0]$ & 3 & 0 & 3 & simple: $\mathfrak{s l}_{2}$ & $(3)$ \\
\hline
\end{tabular}

The listed dimensions do not separate types [1111] and [211], as well as [22] and [31]. Yet, the symmetry algebras do distinguish between them. To see this let $\mathbf{z}=\sum_{i=1}^{8} z_{i} e_{i}$ be a general element of $\mathfrak{s}=\left\langle e_{1}, \ldots, e_{8}\right\rangle$ in the first two cases. Denote by $\operatorname{ad}_{\mathbf{z}} \in \operatorname{End}(\mathfrak{s})$ the adjoint operator. For the Segre type [1111] its spectrum is $\operatorname{Sp}\left(\operatorname{ad}_{\mathbf{z}}\right)=\left\{0(\times 4), z_{1}, z_{2}, z_{3}, z_{4}\right\}$, while for the Segre type [211] it is $\operatorname{Sp}\left(\operatorname{ad}_{\mathbf{z}}\right)=\left\{0(\times 4), z_{1}(\times 2), z_{2}, z_{3}\right\}$. Thus multiplicities of the eigenvalues for general $\mathbf{z}$ distinguish these cases.

However the other two types are not distinguished by the multiplicities. Here $\operatorname{dim} \mathfrak{s}=9$, so let $\mathbf{z}=\sum_{i=1}^{9} z_{i} e_{i}$. For the Segre type [22] we have $\operatorname{Sp}\left(\operatorname{ad}_{\mathbf{z}}\right)=\left\{0(\times 3), \pm i z_{1}, z_{2}, z_{3}, z_{1}+z_{2}, z_{3}-z_{1}\right\}$, and for the Segre type [31], $\operatorname{Sp}\left(\operatorname{ad}_{\mathbf{z}}\right)=\left\{0(\times 3), z_{1}, z_{2}, z_{3}, 2 z_{2}, z_{1}+z_{2}, z_{1}+2 z_{2}\right\}$. But since linear relations among the eigenvalues in these two cases are different, these types are also distinguished by the symmetry algebras.

\section{Proofs of the main results}

After a short remark on the action of SL(6), we investigate the differential prolongation of conditions of linear degeneracy (10). The main feature of this second-order PDE system is its non-involutivity, manifesting itself in additional (hidden) second-order differential constraints. These constraints are obtained by differentiations and linear combinations of the equations in the original system. Afterwards, we complete the proof of Theorem 1.

\subsection{Action of the equivalence group}

While the action of $\mathbf{S L}(6)$ on the Grassmannian $\mathbf{G r}(4,6)$ is transitive, the action on its tangent space $T \mathbf{G r}(4,6)$ has orbits distinguished by the rank of the corresponding $2 \times 4$ matrices. We will need the action on the space of 1-jets $J_{6}^{1} \mathbf{G r}(4,6)$ of submanifolds $X \subset \mathbf{G r}(4,6)$ of dimension 6 , which can be identified with the space $\mathbf{G r}_{6}(T \mathbf{G r}(4,6))$ locally isomorphic to $J^{1}\left(\mathbb{R}^{6}, \mathbb{R}^{2}\right)$.

Lemma. The equivalence group $\mathbf{S L}(6)$ has a unique Zariski open orbit in the space $J_{6}^{1} \mathbf{G r}(4,6)$ (its complement consists of 1-jets of degenerate systems).

Proof. The stabilizer in $\mathbf{S L}(6)$ of a point $o \in \mathbf{G r}(4,6)$ is the parabolic subgroup $P_{o}=S(\mathbf{G L}(2) \times$ $\mathbf{G L}(4)) \ltimes\left(\mathbb{R}^{2} \otimes \mathbb{R}^{4}\right)$ of upper-triangular block matrices of the size $2+4$. The summand $\mathbb{R}^{2} \otimes \mathbb{R}^{4}$ acts 
trivially on $T_{o} \mathbf{G r}(4,6)$, so the effective action is only supported by the subgroup $S(\mathbf{G L}(2) \times$ $\mathbf{G L}(4))$. It is easy to check that this action is transitive on 6-planes corresponding to nondegenerate 1 -jets of $X$ characterised by $\operatorname{det} g \neq 0$ where $g$ denotes a metric representative of the canonical conformal structure $[g]$ (see Section 1.2).

At the level of Lie algebra $\mathfrak{s l}(6)$, the prolongation of the 35 infinitesimal generators $\mathbf{U}_{i}, \mathbf{V}_{j}$, $\mathbf{X}_{i j}, \mathbf{L}_{i j}, \mathbf{P}_{i}, \mathbf{Q}_{j}$ (see Section 1.4) to $J^{1}\left(\mathbb{R}^{6}, \mathbb{R}^{2}\right)$ has full rank in the Zariski open set of nondegenerate 1-jets. Indeed, the $35 \times 20$ matrix of coefficients of these vector fields drops rank precisely on the submanifold $\operatorname{det} g=0$.

Remark. The next Sections contain details of calculations assisted with symbolic packages Maple and Mathematica. However, even these packages cannot resolve the large linear systems that arise after a prolongation to higher (third, forth and fifth) jets. To handle this difficulty we used the following trick: since $\mathbf{S L}(6)$ acts on $J^{1}\left(\mathbb{R}^{6}, \mathbb{R}^{2}\right)$ with an open orbit consisting precisely of admissible 1-jets, and since the prolongation, involutivity and integrability are $\mathbf{S L}(6)$-equivariant properties, we can substitute any numerical non-degenerate 1-jet into all prolonged equations; we used $\left(f_{1}, f_{2}, f_{3}, f_{4}, f_{5}, f_{6}\right)=(0,1,0,1,0,0),\left(h_{1}, h_{2}, h_{3}, h_{4}, h_{5}, h_{6}\right)=(0,0,1,0,0,0)$. This allows to resolve the arising systems, and to compute their ranks without any loss of generality.

\subsection{Prolongation of the conditions of linear degeneracy}

To describe the result we will exploit the language of formal theory of differential equations, cf. [19]. Recall that a system of PDEs of order $k$ on sections of a bundle $\nu$ over a manifold $X$ can be respresented as a submanifold $\mathcal{E}_{k} \subset J^{k}(\nu)$ in the space of jets. In our case, $X \subset \mathbf{G r}(4,6)$ is the sixfold encoding the system, and $\nu=T_{X} \mathbf{G r}(4,6) / T X$ is its normal bundle. Locally, in the affine chart we can identify $X=\mathbb{R}^{6}\left(u_{1}, u_{2}, u_{3}, v_{1}, v_{2}, v_{3}\right)$ and $\nu=X \times \mathbb{R}^{6}\left(u_{4}, v_{4}\right)$ with sections given by (9). Thus, an affine chart of $J^{k}(\nu)$ is the space $J^{k}\left(\mathbb{R}^{6}, \mathbb{R}^{2}\right)$ of jets of maps $(f, h): \mathbb{R}^{6} \rightarrow \mathbb{R}^{2}$, and we will further denote this space by $J^{k}$.

Let us consider the system $\mathcal{E}_{2} \subset J^{2}$ given by 20 PDEs (10) (note that these equations, $E_{l}=0$, are quadratic expressions that are linear in 2-jets with coefficients being linear in 1jets). Its prolongation $\mathcal{E}_{3}=\mathcal{E}_{2}^{(1)} \subset J^{3}$ is given by adding $20 \cdot 6=120$ equations obtained by differentiating (10) (note that higher-order terms of these equations, $D_{i} E_{l}=0$, are linear in 3 -jets with coefficients being linear in 1-jets).

These equations however are not in the Frobenius (closed) form, meaning that not all 3-jets, which are fibre variables of the bundle $\pi_{3,2}: J^{3} \rightarrow J^{2}$ of rank $2 \cdot\left(\begin{array}{c}6+2 \\ 3\end{array}\right)=112$, can be expressed in terms of lower-order jets. In fact, the number of free 3-jets at this step is 17 (invariantly, this means that the symbol $g_{3}=\operatorname{Ker}\left(d \pi_{3,2}: T \mathcal{E}_{3} \rightarrow T \mathcal{E}_{2}\right) \subset S^{3} \mathbb{R}^{6 *} \otimes \mathbb{R}^{2}$ has codimension 17$)$, whence $120-(112-17)=25$ combinations of our equations have vanishing 3 -symbols. These equations of order 2 define a proper locus $\tilde{\mathcal{E}}_{2}:=\pi_{3,2}\left(\mathcal{E}_{3}\right) \subset \mathcal{E}_{2}$ given by a quadratic ideal in 2-jet variables.

Proposition 5. The system $\tilde{\mathcal{E}}_{2}=\tilde{\mathcal{E}}_{2}^{\prime} \cup \tilde{\mathcal{E}}_{2}^{\prime \prime}$ is a reducible algebraic (sub-) variety in $J^{2}$ with an irreducible component $\tilde{\mathcal{E}}_{2}^{\prime}$ of codimension 24 and an irreducible component $\tilde{\mathcal{E}}_{2}^{\prime \prime}$ of codimension 30. The intersection $\tilde{\mathcal{E}}_{2}^{\prime} \cap \tilde{\mathcal{E}}_{2}^{\prime \prime}$ is an irreducible algebraic variety of codimension 34.

Proof. This is obtained by prime ideal decomposition. Indeed, the substitution of a nondegenerate 1-jet $x_{1}=\left\{\left(f_{a}, h_{b}\right)\right\}$ into the equations (see Remark in Section 3.1) splits the system into 20 linear, and a bunch of quadratic equations in the variables $f_{a b}, h_{a b}, 1 \leq a \leq b \leq 6$. The quadratic ideal is then seen to be generated by products of linear expressions (from the set of 4 
and 10 equations respectively), so that its locus in every $\pi_{2,1}^{-1}\left(x_{1}\right)$ is the union of two subspaces that are linear in 2-jet variables (but polynomial in $x_{1} \in J^{1}$ ), and this implies the claim.

The second prolongation $\mathcal{E}_{4}=\mathcal{E}_{2}^{(2)}=\mathcal{E}_{3}^{(1)} \subset J^{4}$ (obtained by adding equations $D_{i} D_{j} E_{l}=0$ whose higher-order terms are linear in 4 -jets with coefficients being linear in 1-jets) is already in the Frobenius form (all 4-jets are expressed in terms of lower-order jets).

Yet the system generated by $\tilde{\mathcal{E}}_{2}$ is not in involution: the prolongation $\tilde{\mathcal{E}}_{2}^{(1)} \subset J^{3}$ is not in closed form - the number of free 3-jets is 8 . Even the system $\pi_{4,3}\left(\mathcal{E}_{4}\right) \subset J^{3}$ is not closed the number of free 3 -jets at this step is 3 . We have to do one more prolongation: for the system $\mathcal{E}_{5}=\mathcal{E}_{2}^{(3)}=\mathcal{E}_{4}^{(1)} \subset J^{5}$ (obtained by adding equations $D_{i} D_{j} D_{k} E_{l}=0$ ) the projection $\tilde{\mathcal{E}}_{3}=\pi_{5,3}\left(\mathcal{E}_{5}\right) \subset J^{3}$ is Frobenius (all 3-jets can be expressed, or equivalently the symbol $\tilde{g}_{3}=0$ ).

Consequently, we obtain a PDE system $\tilde{\mathcal{E}}$ given by the second-order equation-manifold $\tilde{\mathcal{E}}_{2}$, the third-order locus $\tilde{\mathcal{E}}_{3}$ (obtained by adding 112 third-order PDE), and its prolongations.

Proposition 6. The system $\tilde{\mathcal{E}}$ is involutive.

Proof: Due to Proposition 5 this system splits as $\tilde{\mathcal{E}}=\tilde{\mathcal{E}}^{\prime} \cup \tilde{\mathcal{E}}^{\prime \prime}$ into the union of systems that are linear in jets of order $>1$. The symbols $\tilde{g}_{k}=\operatorname{Ker}\left(d \pi_{k, k-1}: T \tilde{\mathcal{E}}_{k} \rightarrow T \tilde{\mathcal{E}}_{k-1}\right)$ of the new systems satisfy: $\operatorname{dim} \tilde{g}_{0}=2, \operatorname{dim} \tilde{g}_{1}=2 \cdot 6=12, \operatorname{dim} \tilde{g}_{2}^{\prime}=42-24=18, \operatorname{dim} \tilde{g}_{2}^{\prime \prime}=42-30=12, \operatorname{dim} \tilde{g}_{k}=0$ for $k>2$. Thus, the solution spaces of these equations have dimensions that are bounded by $\operatorname{dim} \tilde{g}_{0}+\operatorname{dim} \tilde{g}_{1}+\operatorname{dim} \tilde{g}_{2}^{\prime}=32$ and $\operatorname{dim} \tilde{g}_{0}+\operatorname{dim} \tilde{g}_{1}+\operatorname{dim} \tilde{g}_{2}^{\prime \prime}=26$, respectively.

To ensure involutivity we have to check that for every point $o \in X$ and every $\infty$-jet admissible by the system $\tilde{\mathcal{E}}_{o}=\tilde{\mathcal{E}} \cap \pi_{\infty, 0}^{-1}(o)$ over it, there is a solution to (10) with this jet at $o$.

Let us start with the system $\tilde{\mathcal{E}}^{\prime}$. We claim that all its solutions are given by the Chasles construction. The latter have normal forms specified in Tables 2-6. The most general solution has Segre type [0] and since its stabilizer in $\mathbf{S L}(6)$ is 3-dimensional, the space of solutions of the Chasles type has dimension $35-3=32$.

Another way to see this is as follows. The general solution of the Chasles type is given by the 2-planes $\langle A, B\rangle \in \mathbf{G r}(2, U)$, where $U=\operatorname{Hom}(W, V) \simeq \mathbb{R}^{6} \otimes \mathbb{R}^{7 *}$ is the space of $6 \times 7$ matrices: $X_{A, B}=\{A x \wedge B x: x \in W\} \subset \mathbf{G r}(2, V)$. Reparametrization $(A, B) \sim(P A, P B)$ yields the same solution for $P \in \mathbf{S L}(7)$ (more general equivalence $(A, B) \sim(P A Q, P B Q)$ yields equivalent manifolds $\left.X_{A, B}\right)$. Thus the space of solutions of the Chasles type has dimension $80-48=32$.

Moreover, the map $\langle A, B\rangle \mapsto j_{o}^{\infty}\left(X_{A B}\right) \in \tilde{\mathcal{E}}_{o}^{\prime}$ from the projective variety $\operatorname{Gr}(2, U)$ to the irreducible variety $\tilde{\mathcal{E}}_{o}^{\prime}$ has an open image (by what we have already computed) and therefore must be epimorphic. This proves the claim about $\tilde{\mathcal{E}}^{\prime}$.

For the system $\tilde{\mathcal{E}}^{\prime \prime}$ we claim that all solutions are sixfolds $X$ of the Monge-Ampère type. The normal forms are collected in Table 1 and the most general of those is the Husain equation. Since its stabilizer with respect to $\mathbf{S L}(6)$ is 9-dimensional, the space of 2-component Monge-Ampère systems has dimension $35-9=26$. We can show that all solutions of $\tilde{\mathcal{E}}^{\prime \prime}$ are Monge-Ampère by an approach similar to the case of $\tilde{\mathcal{E}}^{\prime}$, but it is easier to conclude the claim by observing that 30 second-order equations specifying $\tilde{\mathcal{E}}_{2}^{\prime \prime}$ are exactly the PDEs from Proposition 3.

Finally, the intersection $\tilde{\mathcal{E}}^{\prime} \cap \tilde{\mathcal{E}}^{\prime \prime}$ consists of linearizable systems. Indeed, the stabilizer of a linear system is a 13-dimensional subgroup of $\mathbf{S L}(6)$, so that the space of such systems has dimension $35-13=22$, which coincides with $\operatorname{dim} \tilde{g}_{0}+\operatorname{dim} \tilde{g}_{1}+\operatorname{dim}\left(\tilde{g}_{2}^{\prime} \cap \tilde{g}_{2}^{\prime \prime}\right)=2+12+8$.

We can summarize the prolongation-projection of the conditions of linear degeneracy in the following diagram. 


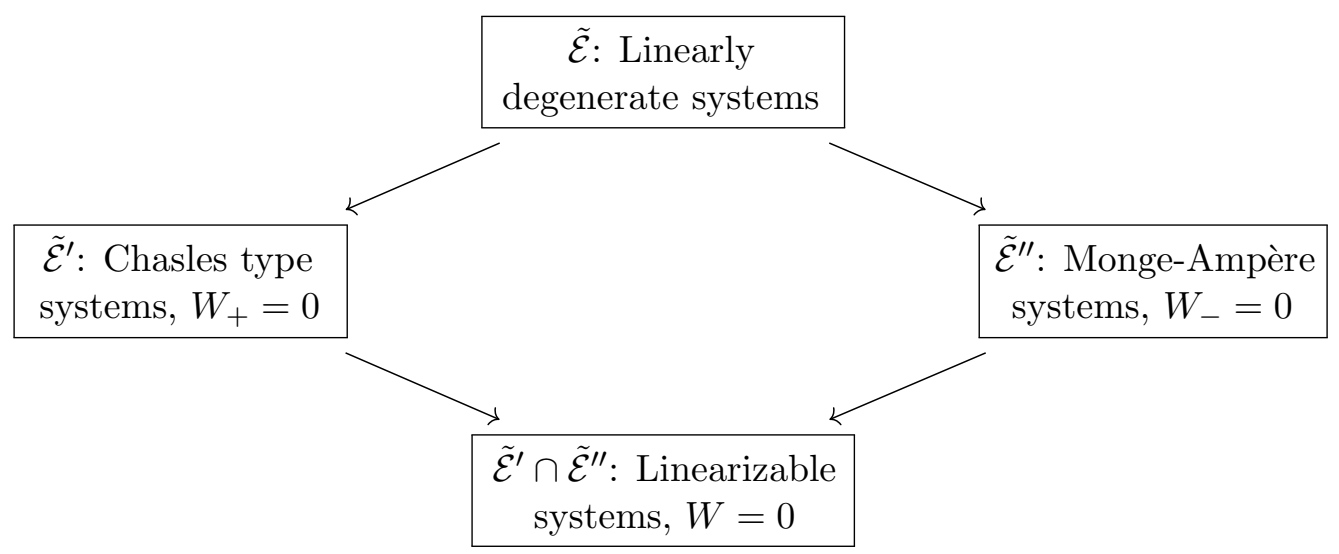

Note that the two irreducible components can be characterised in terms of the Weyl tensor of the canonical conformal structure as self-dual and anti-self-dual systems (up to the change of orientation).

\subsection{Proof of Theorem 1}

Implication (a) $\Longrightarrow(\mathbf{c})$. Our strategy is to derive a set of constraints for the right-hand sides $f$ and $h$ in (9) that are necessary and sufficient for integrability. As outlined in [7], in three dimensions this leads to an involutive system of third-order integrability conditions for $f$ and $h$. The crucial difference occuring in the $4 \mathrm{D}$ case is the appearance, along with third-order constraints, of a whole set of second-order integrability conditions that turn out to be equivalent to relations (10) characterising linearly degenerate systems. This shows that the requirement of integrability in higher dimensions is far more rigid. Here are the details of calculations. Based on evolutionary representation (9) we introduce the notation

$$
u_{1}=a, u_{2}=b, u_{3}=c, v_{1}=p, v_{2}=q, v_{3}=r, u_{4}=f(a, b, c, p, q, r), v_{4}=h(a, b, c, p, q, r) .
$$

This results in the equivalent quasilinear representation of type (5),

$$
\begin{gathered}
a_{2}=b_{1}, \quad a_{3}=c_{1}, \quad a_{4}=f(a, b, c, p, q, r)_{1}, \\
b_{3}=c_{2}, \quad b_{4}=f(a, b, c, p, q, r)_{2}, \quad c_{4}=f(a, b, c, p, q, r)_{3}, \\
p_{2}=q_{1}, \quad p_{3}=r_{1}, \quad p_{4}=h(a, b, c, p, q, r)_{1}, \\
q_{3}=r_{2}, \quad q_{4}=h(a, b, c, p, q, r)_{2}, \quad r_{4}=h(a, b, c, p, q, r)_{3} .
\end{gathered}
$$

Following the method of hydrodynamic reductions let us look for multi-phase solutions where $a, b, c, p, q, r$ are sought as functions of $N$ phases $R^{1}, \ldots, R^{N}$ that are required to satisfy a triple of consistent $(1+1)$-dimensional systems $(6)$,

$$
R_{x^{2}}^{i}=\mu^{i}(R) R_{x^{1}}^{i}, \quad R_{x^{3}}^{i}=\eta^{i}(R) R_{x^{1}}^{i}, \quad R_{x^{4}}^{i}=\lambda^{i}(R) R_{x^{1}}^{i} .
$$

Here the characteristic speeds $\mu^{i}, \eta^{i}$ and $\lambda^{i}$ satisfy the commutativity conditions (7),

$$
\frac{\partial_{j} \lambda^{i}}{\lambda^{j}-\lambda^{i}}=\frac{\partial_{j} \mu^{i}}{\mu^{j}-\mu^{i}}=\frac{\partial_{j} \eta^{i}}{\eta^{j}-\eta^{i}}
$$


$i \neq j, \partial_{j}=\partial_{R^{j}}$. The substitution into (16) implies the relations

$$
\partial_{i} b=\mu^{i} \partial_{i} a, \quad \partial_{i} c=\eta^{i} \partial_{i} a, \quad \partial_{i} q=\mu^{i} \partial_{i} p, \quad \partial_{i} r=\eta^{i} \partial_{i} p,
$$

as well as

$$
\begin{aligned}
\left(\lambda^{i}-f_{a}-\mu^{i} f_{b}-\eta^{i} f_{c}\right) \partial_{i} a & =\left(f_{p}+\mu^{i} f_{q}+\eta^{i} f_{r}\right) \partial_{i} p \\
\left(\lambda^{i}-h_{p}-\mu^{i} h_{q}-\eta^{i} h_{r}\right) \partial_{i} p & =\left(h_{a}+\mu^{i} h_{b}+\eta^{i} h_{c}\right) \partial_{i} a .
\end{aligned}
$$

The last two equations imply the dispersion relation connecting $\lambda^{i}, \mu^{i}$ and $\eta^{i}$,

$$
\left(\lambda^{i}-f_{a}-\mu^{i} f_{b}-\eta^{i} f_{c}\right)\left(\lambda^{i}-h_{p}-\mu^{i} h_{q}-\eta^{i} h_{r}\right)=\left(f_{p}+\mu^{i} f_{q}+\eta^{i} f_{r}\right)\left(h_{a}+\mu^{i} h_{b}+\eta^{i} h_{c}\right) .
$$

In what follows we assume that the dispersion relation defines a non-degenerate quadric in the $(\lambda, \mu, \eta)$-space: this is equivalent to the requirement of non-degeneracy from Section 1.2. Setting in (19) $\partial_{i} a=\varphi^{i} \partial_{i} p$ we can parametrise $\mu^{i}$ and $\lambda^{i}$ in the form

$$
\begin{gathered}
\mu^{i}=-\frac{f_{p}+\left(f_{a}-h_{p}\right) \varphi^{i}-h_{a} \varphi^{2}+\eta^{i}\left(f_{r}+\left(f_{c}-h_{r}\right) \varphi^{i}-h_{c} \varphi^{i^{2}}\right)}{f_{q}+\left(f_{b}-h_{q}\right) \varphi^{i}-h_{b} \varphi^{i^{2}}}, \\
\lambda^{i}=\frac{\left(f_{q}+f_{b} \varphi^{i}\right)\left(h_{p}+h_{a} \varphi^{i}\right)-\left(f_{p}+f_{a} \varphi^{i}\right)\left(h_{q}+h_{b} \varphi^{i}\right)+\eta^{i}\left[\left(f_{q}+f_{b} \varphi^{i}\right)\left(h_{r}+h_{c} \varphi^{i}\right)-\left(f_{r}+f_{c} \varphi^{i}\right)\left(h_{q}+h_{b} \varphi^{i}\right)\right]}{f_{q}+\left(f_{b}-h_{q}\right) \varphi^{i}-h_{b} \varphi^{2}} .
\end{gathered}
$$

Substituting these expressions into commutativity conditions (17), and using the relations

$$
\partial_{i} a=\varphi^{i} \partial_{i} p, \quad \partial_{i} b=\mu^{i} \varphi^{i} \partial_{i} p, \quad \partial_{i} c=\eta^{i} \varphi^{i} \partial_{i} p, \quad \partial_{i} q=\mu^{i} \partial_{i} p, \quad \partial_{i} r=\eta^{i} \partial_{i} p,
$$

we obtain $\partial_{j} \varphi^{i}$ and $\partial_{j} \eta^{i}$ in the form $\partial_{j} \varphi^{i}=(\ldots) \partial_{j} p, \partial_{j} \eta^{i}=(\ldots) \partial_{j} p, i \neq j$, where dots denote rational expressions in $\varphi^{i}, \varphi^{j}, \eta^{i}, \eta^{j}$ whose coefficients depend on second-order partial derivatives of $f$ and $h$. Calculating consistency conditions for relations (20) we obtain (one and the same!) expression for $\partial_{i} \partial_{j} p$ in the form $\partial_{i} \partial_{j} p=(\ldots) \partial_{i} p \partial_{j} p, i \neq j$, where, again, dots denote terms rational in $\varphi^{i}, \varphi^{j}, \eta^{i}, \eta^{j}$. Ultimately, $N$-phase solutions are governed by the relations

$$
\partial_{j} \varphi^{i}=(\ldots) \partial_{j} p, \quad \partial_{j} \eta^{i}=(\ldots) \partial_{j} p, \quad \partial_{i} \partial_{j} p=(\ldots) \partial_{i} p \partial_{j} p,
$$

$i \neq j$. Direct calculation of the compatibility conditions based on (20) and (21) results in

$$
\begin{gathered}
\partial_{k} \partial_{j} \varphi^{i}-\partial_{j} \partial_{k} \varphi^{i}=(\ldots) \partial_{j} p \partial_{k} p, \quad \partial_{k} \partial_{j} \eta^{i}-\partial_{j} \partial_{k} \eta^{i}=(\ldots) \partial_{j} p \partial_{k} p \\
\partial_{k} \partial_{j} \partial_{i} p-\partial_{j} \partial_{k} \partial_{i} p=(\ldots) \partial_{i} p \partial_{j} p \partial_{k} p
\end{gathered}
$$

where dots denote complicated rational expressions in $\varphi^{i}, \varphi^{j}, \varphi^{k}$ and $\eta^{i}, \eta^{j}, \eta^{k}$, whose coefficients depend on partial derivatives of $f$ and $h$ up to the third order. To ensure the solvability of equations (21) we set all these coefficients equal to zero. Without any loss of generality we can set $(i, j, k)=(1,2,3)$. In particular, the coefficient in the numerator of $\partial_{3} \partial_{2} \partial_{1} p-\partial_{2} \partial_{3} \partial_{1} p$ at the monomial $\left(\varphi^{1}\right)^{12}\left(\varphi^{2}\right)^{9}\left(\varphi^{3}\right)^{6}\left(\eta^{1}\right)^{6}\left(\eta^{2}\right)\left(\eta^{3}\right)^{3}$ has the form $\tau L^{2}$ where $\tau$ is a nonzero expression depending on first-order derivatives of $f$ and $h$ only, and

$$
\begin{aligned}
L= & \left(f_{r} h_{b}-f_{q} h_{c}\right)\left(f_{r}^{2} f_{q q}-2 f_{q} f_{r} f_{q r}+f_{q}^{2} f_{r r}\right) \\
& +\left(f_{c} f_{q}-f_{b} f_{r}+f_{r} h_{q}-f_{q} h_{r}\right)\left(f_{r}^{2} h_{q q}-2 f_{q} f_{r} h_{q r}+f_{q}^{2} h_{r r}\right) .
\end{aligned}
$$

The condition $L=0$ is linear in the second-order derivatives of $f$ and $h$. Let us now utilise the fact that conditions of integrability must be invariant under the action of the equivalence 
group. Acting on the condition $L=0$ by transformations from the equivalence group $\mathbf{S L}(6)$ we obtain all of the 20 second-order conditions of linear degeneracy (10).

Implications $(\mathbf{b}) \Longrightarrow(\mathbf{c})$. Let $[g]$ be the conformal structure defined by the characteristic variety of system (1). We shall demonstrate that, with a proper choice of orientation, the condition of conformal half-flatness implies linear degeneracy. Let us note that in the splitting of the Weyl tensor, $W=W_{+}+W_{-}$, we use the Hodge star operator which depends on the square root of

$$
\begin{aligned}
\operatorname{det} g=\left(\frac { 1 } { 4 } \left(f_{a} f_{q} h_{c}-\right.\right. & f_{a} f_{r} h_{b}-f_{b} f_{p} h_{c}+f_{b} f_{r} h_{a}+f_{c} f_{p} h_{b} \\
& \left.\left.\quad-f_{c} f_{q} h_{a}-f_{p} h_{b} h_{r}+f_{p} h_{c} h_{q}+f_{q} h_{a} h_{r}-f_{q} h_{c} h_{p}-f_{r} h_{a} h_{q}+f_{r} h_{b} h_{p}\right)\right)^{2},
\end{aligned}
$$

in the notation of (16). Choosing $\sqrt{\operatorname{det} g}$ to be the expression in big parentheses, we define the tensor $* W$ and observe the following. The condition of self-duality, $W_{-}=0$, consists of 30 equations that are equivalent to those of Proposition 3, and characterise PDEs of Monge-Ampère type given by the system $\tilde{\mathcal{E}}^{\prime \prime}$ from Section 3.2. The condition of anti-self-duality, $W_{+}=0$, consists of 24 equations that characterise general linearly degenerate PDEs associated with quadratic maps $\mathbb{P}^{6} \rightarrow \mathbf{G r}(4,6)$. These are given by the system $\tilde{\mathcal{E}}^{\prime}$ from Section 3.2.

Implication $(\mathbf{c}) \Longrightarrow(\mathbf{a})$. Since all linearly degenerate equations are classified in Tables 1-6, it is straightforward to check that each of them passes the test for hydrodynamic integrability.

Implication $(\mathbf{c}) \Longrightarrow(\mathbf{b})$. Again, linearly degenerate equations have normal forms represented in Tables 1-6. It can be straightforwardly verified that conformal structures corresponding to them are half-flat $(* W= \pm W)$ on every solution.

More conceptually, the result can be seen as follows. Every equation in Tables 1-6 has a Lax pair with a spectral parameter, and according to [3] this implies self-duality (with a proper choice of orientation). Here is a brief explanation. This Lax pair is a 2-distribution on the correspondence space $\left(x^{1}, \ldots, x^{4}, \lambda\right)$. The integral surfaces of this distribution projected to the $\mathbf{x}$-space form a 3-parametric family of null totally geodesic surfaces with respect to the conformal structure on every solution $u=u(\mathbf{x}), v=v(\mathbf{x})$. According to Penrose [23], the existence of such surfaces (known as $\alpha$-surfaces) is equivalent to self-duality.

Implications $(\mathbf{d}) \Longleftrightarrow(\mathbf{c})$. This is a direct corollary of Section 3.2.

This finishes the proof of Theorem 1.

Remark. Geometrically, Theorem 1 can be interpreted as follows. Let $X$ be a sixfold in $\operatorname{Gr}(4,6)$. Taking a point $o \in X$ and projectivising the intersection of the tangent space $\mathrm{T}_{o} X$ with the Serge cone $C$ in $\mathrm{T}_{o} \mathbf{G r}(4,6)$, which is the cone over a non-singular rational fourfold of degree four in $\mathbb{P}^{7}=\mathbb{P} T_{o} \mathbf{G r}(4,6)$, one obtains a rational surface of degree four. This surface, known as a rational normal scroll, can be interpreted as the set of matrices of rank one in the tangent space $\mathrm{T}_{o} X$ (recall that $\mathrm{T}_{o} \mathbf{G r}(4,6)$ is identified with the space of $2 \times 4$ matrices: here we utilise the duality between $\mathbf{G r}(4,6)$ and $\mathbf{G r}(2,6))$. Thus, the projectivised tangent bundle of $X$ is equipped with a field of rational normal scrolls of degree four. The integrability conditions can be reformulated as the requirement of the existence in $X$ of infinitely many holonomic trisecant threefolds whose projectivised tangent spaces intersect the rational normal scroll at three distinct points. These threefolds correspond to three-component hydrodynamic reductions (we refer to $[7,26]$ for a related discussion). Theorem 1 states that this requirement forces $X$ to be algebraic, more precisely, $X$ must be either a codimension 2 linear section of the Plücker embedding $\mathbf{G r}(4,6) \hookrightarrow \mathbb{P}^{14}$, or the image of a quadratic map $\mathbb{P}^{6} \rightarrow \mathbf{G r}(4,6)$. It would be interesting to have a purely geometric proof of this result. 


\section{Concluding remarks}

We have obtained a complete description of integrable systems associated with sixfolds in $\operatorname{Gr}(4,6)$. The corresponding sixfolds are either codimension two linear sections of the Plücker embedding $\mathbf{G r}(4,6) \hookrightarrow \mathbb{P}^{14}$ or images of quadratic maps $\mathbb{P}^{6} \rightarrow \mathbf{G r}(4,6)$. Conversely, every sixfold of one of the above types gives rise to an integrable system.

Let us compare the case of PDE systems in 3D associated to fourfolds in $\mathbf{G r}(3,5)$ studied in [7] to that of PDE systems in 4D studied in this paper. While the main theorems expressing integrability of the systems $\mathcal{E}=\{F=0, H=0\}$ via the geometric properties of solutions (Einstein-Weyl property in 3D and self-duality in 4D) are similar in spirit, there are several important differences.

\begin{tabular}{|c|c|}
\hline Integrable systems in $3 \mathrm{D}$ & Integrable systems in $4 \mathrm{D}$ \\
\hline The parameter space $\mathcal{M}=\mathcal{M}^{30}$ is irreducible & The parameter space $\mathcal{M}=\mathcal{M}_{1}^{26} \cup \mathcal{M}_{2}^{32}$ is reducible \\
\hline The generic equation $\mathcal{E} \in \mathcal{M}$ is transcendental & The generic equation $\mathcal{E} \in \mathcal{M}$ is algebraic \\
\hline $\begin{array}{l}\text { The moduli space } \mathcal{M} / G \text { is a rational } \\
\text { algebraic variety of positive dimension }(=6)\end{array}$ & $\begin{array}{l}\text { The moduli space } \mathcal{M} / G \text { is } 0 \text {-dimensional } \\
\text { Each component of } \mathcal{M} \text { has a unique Zariski open orbit }\end{array}$ \\
\hline Parametrizable by special functions & Finite classification of integrable cases \\
\hline There exists $\partial_{\lambda}$ term in the Lax pair (in general) & There is no $\partial_{\lambda}$ term in the Lax pair (hyper-complex case) \\
\hline
\end{tabular}

Finally, it would also be interesting to investigate the integrability problem for Grassmannians of higher dimensions. For instance, let $u, v, w$ be functions of the independent variables $x^{1}, \ldots, x^{4}$. A first-order 3-component system,

$$
F_{i}\left(u_{1}, \ldots, u_{4}, v_{1}, \ldots, v_{4}, w_{1}, \ldots, w_{4}\right)=0, \quad i=1,2,3,
$$

is naturally associated with a codimension 3 submanifold $X \subset \mathbf{G r}(4,7)$. We conjecture that the requirement of integrability forces $X$ to be either a codimension 3 linear section of $\mathbf{G r}(4,7)$ (the case of Monge-Ampère systems), or the image of a cubic map $\mathbb{P}^{9} \rightarrow \mathbf{G r}(4,7)$ (general linearly degenerate systems). The results of [6] suggest that even under these restrictions the corresponding systems will not automatically be integrable, and additional geometric restrictions on $X$ will be required.

\section{Acknowledgements}

We thank A. Bolsinov, I. Dolgachev, E. Mezzetti, M. Pavlov and A. Prendergast-Smith for clarifying discussions. We also thank the LMS for their support of BD to Loughborough making this collaboration possible. BK is grateful to the Universities of Loughborough and Cambridge for hospitality. The research of EVF was partially supported by the EPSRC grant EP/N031369/1.

\section{References}

[1] L.V. Bogdanov, B.G. Konopelchenko, On the $\bar{\partial}$-dressing method applicable to heavenly equation, Phys. Lett. A 345, no. 1-3 (2005), 137-143.

[2] G. Boillat, Sur la forme générale du système de Monge-Ampère, C. R. Acad. Sci. Paris Sér. I Math. 325, no. 3 (1997), 339-342. 
[3] D.M.J. Calderbank, B. Kruglikov, Integrability via geometry: dispersionless differential equations in three and four dimensions, arXiv:1612.02753.

[4] M. Chasles, Considérations sur la méthode générale exposée dans la séance du 15 Février, Comptes Rendus 58 (1864) 1167-76.

[5] I.V. Dolgachev, Classical algebraic geometry. A modern view, Cambridge University Press, Cambridge (2012), 639 pp.

[6] B. Doubrov, E.V. Ferapontov, On the integrability of symplectic Monge-Ampère equations, J. Geom. Phys. 60 (2010), 1604-1616.

[7] B. Doubrov, E.V. Ferapontov, B. Kruglikov, V.S. Novikov, On integrability in Grassmann geometries: integrable systems associated with fourfolds in $\operatorname{Gr}(3,5)$, arXiv:1503.02274.

[8] B. Doubrov, E.V. Ferapontov, B. Kruglikov, V. Novikov, On a class of integrable systems of Monge-Ampère type, arXiv:1701.02270; to appear in J. Math. Phys. (2017).

[9] M. Dunajski, The twisted photon associated to hyper-Hermitian four-manifolds, J. Geom. Phys. 30, no. 3 (1999), 266-281.

[10] M. Dunajski, E.V. Ferapontov, B.S. Kruglikov, On the Einstein-Weyl and conformal self-duality equations, J. Math. Phys. 56, 083501 (2015).

[11] E.V. Ferapontov, K.R. Khusnutdinova, On the integrability of (2+1)-dimensional quasilinear systems, Comm. Math. Phys. 248 (2004), 187-206.

[12] E.V. Ferapontov, M.V. Pavlov, Hydrodynamic reductions of the heavenly equation, Class. Quantum Grav. 20 (2003), 2429-2441.

[13] E.V. Ferapontov, K.R. Khusnutdinova, Hydrodynamic reductions of multi-dimensional dispersionless PDEs: the test for integrability, J. Math. Phys. 45 (2004), 2365-2377.

[14] E.V. Ferapontov, K.R. Khusnutdinova, C. Klein, On linear degeneracy of integrable quasilinear systems in higher dimensions, Lett. Math. Phys. 96, no. 1 (2011), 5-35.

[15] E.V. Ferapontov, B.S. Kruglikov, Dispersionless integrable systems in $3 D$ and Einstein-Weyl geometry, J. Diff. Geom. 97 (2014), 215-254.

[16] F.R. Gantmacher, The theory of matrices, vol. 1-2, Chelsea Publishing Co., New York (1959).

[17] J. Gibbons, S.P. Tsarev, Reductions of the Benney equations, Phys. Lett. A 211 (1996), 19-24; J. Gibbons, S.P. Tsarev, Conformal maps and reductions of the Benney equations, Phys. Lett. A 258 (1999), 263-270.

[18] V. Husain, Self-dual gravity as a two-dimensional theory and conservation laws, Class. Quantum Grav. 11, no. 4 (1994), 927-937.

[19] B. Kruglikov, V. Lychagin, Geometry of differential equations, Handbook of Global Analysis, Eds. D. Krupka, D. Saunders, 727-773, Elsevier (2007).

[20] S.V. Manakov, P.M. Santini, Inverse scattering problem for vector fields and the Cauchy problem for the heavenly equation, Phys. Lett. A 359, no. 6 (2006), 613-619.

[21] S.V. Manakov, P.M. Santini, On the solutions of the second heavenly and Pavlov equations, J. Phys. A 42 , no. 40 (2009), 404013, 11 pp.

[22] A.V. Odesskii, V.V. Sokolov, Integrable pseudopotentials related to generalized hypergeometric functions, Selecta Math. 16 (2010), 145.

[23] R. Penrose, Nonlinear gravitons and curved twistor theory, General Relativity and Gravitation 7, no. 1 (1976), 31-52.

[24] J.F. Plebański, Some solutions of complex Einstein equations, J. Math. Phys. 16 (1975) 2395-2402.

[25] B.L. Rozdestvenskii, A.D. Sidorenko, On the impossibility of 'gradient catastrophe' for weakly nonlinear systems, Z. Vycisl. Mat. i Mat. Fiz. 7 (1967), 1176-1179.

[26] A.D. Smith, A geometry for second-order PDEs and their integrability, Part 1, arXiv:1010.6010v1.

[27] S.P. Tsarev, Poisson brackets and one-dimensional Hamiltonian systems of hydrodynamic type, Soviet Math. Dokl. 31 (1985), 488-491; S.P. Tsarev, The geometry of Hamiltonian systems of hydrodynamic type. The generalized hodograph method, Math. USSR Izvestiya 37 (1991), 397-419.

[28] I. Zakharevich, Nonlinear wave equation, nonlinear Riemann problem, and the twistor transform of Veronese webs, arXiv:math-ph/0006001. 